\title{
Analytic approach to calculations of mass spectra and decay constants of heavy-light quarkonia in the framework of Bethe-Salpeter equation
}

\author{
Eshete Gebrehana, ${ }^{1}$ Shashank Bhatnagar, ${ }^{2, *}$ and Hluf Negash ${ }^{3}$ \\ ${ }^{1}$ Department of Physics, Addis Ababa University, P.O. Box 1176 Addis Ababa, Ethiopia \\ ${ }^{2}$ Department of Physics, University Institute of Sciences, Chandigarh University, Mohali-140413, India \\ ${ }^{3}$ Department of Physics, Samara University, P.O. Box 132, Samara, Ethiopia
}

(Received 23 December 2018; published 26 September 2019)

\begin{abstract}
This work is an extension of the work in [Phys. Rev. D 97, 034021 (2018)] to ground and excited states of $0^{++}, 0^{-+}$, and $1^{--}$of heavy-light $(c \bar{u}, c \bar{s}, b \bar{u}, b \bar{s}$, and $b \bar{c})$ quarkonia in the framework of a QCD motivated Bethe-Salpeter equation (BSE) by making use of the exact treatment of the spin structure $\left(\gamma_{\mu} \otimes \gamma_{\mu}\right)$ in the interaction kernel, in contrast to the approximate treatment of the same in our previous works [H. Negash and S. Bhatnagar, Int. J. Mod. Phys. E 25, 1650059 (2016)., S. Bhatnagar and L. Alemu, Phys. Rev. D 97, 034021 (2018).]). In this $4 \times 4$ BSE framework, the coupled Salpeter equations for $Q \bar{q}$ (that are more involved than the equal mass $(Q \bar{Q})$ mesons) are first shown to decouple for the confining part of interaction, under heavy-quark approximation, and analytically solved, and later the one-gluonexchange interaction is perturbatively incorporated, leading to their mass spectral equations. The analytic forms of wave functions obtained from these equations are then used for calculation of leptonic decay constants of ground and excited states of $0^{-+}$and $1^{--}$as a test of these wave functions and the over all framework.
\end{abstract}

DOI: $10.1103 /$ PhysRevD.100.054034

\section{INTRODUCTION}

During the past few years, there has been a growing interest in the experimental and theoretical studies of heavy-light mesons. This interest arose from the discovery of large $B_{0}-\bar{B}_{0}$ mixing, leading to the hope that $C P$ violation in B-systems may be observed. Further studies on heavy-light mesons are also important for the determination of Cabibo-Kobayashi-Maskawa (CKM) mass matrix elements. These studies on quarkonia need heavy quark dynamics, which can provide a significant test of quantum chromodynamics (QCD). Spectroscopy of heavy quarkonia have been studied through nonperturbative QCD approaches, such as NRQCD [1], QCD sum rule [2], potential models [3-5], lattice QCD [6-8], Bethe-Salpeter equation (BSE) method [9-16], heavy quark effective theory [17], relativistic quantum model (RQM) [18], and chiral perturbation theory [19].

It may be recalled that the discoveries of the low-lying charmonium states and of open-charmed hadrons were

\footnotetext{
*Corresponding author. shashank_bhatnagar@yahoo.com

Published by the American Physical Society under the terms of the Creative Commons Attribution 4.0 International license. Further distribution of this work must maintain attribution to the author(s) and the published article's title, journal citation, and DOI. Funded by SCOAP ${ }^{3}$.
}

instrumental for the acceptance of quarks as truly dynamical entities in general, and of the SM in particular. Thus studies of heavy charmonium $(c \bar{c})$ and bottomonium $(b \bar{b})$ states is a frontier area of research interest. Now, unequal quark heavy meson, $c \bar{b}$ is the only bound state discovered that comprises of two heavy quarks of different flavors, and acts as an intermediate state between $c \bar{c}$ and $b \bar{b}$ states both in mass and size. The discovery of $B_{c}$ state has given a new insight into heavy-quark dynamics, though its vector counterpart, $B_{c} *$, has not yet been discovered in experiments. The quark content of $B_{c}$ forbids its decays into two photons, and can only decay through weak interactions, and have radiative decays, and thus can lead to calculation of CKM matrix elements. The same is true of other heavy-light mesons, $Q \bar{q}(q=u, d, s)$. The dynamics of unequal mass mesons is richer than for $Q \bar{Q}$ mesons, due to the simple fact that for $c \bar{b}$ mesons, the relativistic effects are more important than $c \bar{c}$, since the $c$ quark moves faster in $B_{c}$ meson than in $J / \Psi$ [3].

The renewed interest in recent years in spectroscopy of these heavy and heavy-light hadrons in charm and beauty sectors, which was primarily due to experimental facilities the world over such as BABAR, Belle, CLEO, DELPHI, BES etc. [20-25], have been providing accurate data on these hadrons with respect to their masses and decays. In the process many new states have been discovered such as $\chi_{b 0}(3 P), \chi_{c 0}(2 P), X(3915), X(4260), X(4360), X(4430)$, 
$X(4660)$ [20]. Further, there are also open questions about the quantum number assignments of some of these states such as $X(3915)$ (as to whether it is $\chi_{c 0}(2 P)$ or $\chi_{c 2}(2 P)$ $[26,27])$. Currently there is a lot of excitement about $X Y Z$ particles, that are new charmoniumlike states such as, $Z_{c}(3900), Z_{c}(4020) / Z_{c}(4025)$ [28], which were discovered in BESIII, the states, $Y(4260)[29,30]$ (discovered at $B A B A R$ ), and $X(3872)$ [31] (discovered at Belle). These particles show different features than the conventional charmonium states, and might be good candidates for exotic states. These states might even be hybrid or tetraquark states, or loosely bound charmonium molecules, which are not excluded by QCD.

Thus charmoniumlike states offer us intriguing puzzles. However, since the mass spectrum and the decays of all these bound states can be tested experimentally, theoretical studies on them may throw valuable insight about the heavy quark dynamics. However, owing to the essentially nonperturbative nature of quark dressing in QCD it is impossible to define a "potential" for systems involving quarks lighter than the $b$ quark. And any approach that nevertheless employs a potential for such systems, is unconnected with QCD, and is a potential model. However, such effective models in physics of hadrons are useful as a tool in areas where QCD is not yet able to penetrate.

Now, Bethe-Salpeter equation is basically an eigenvalue problem valid only at the resonance pole, $P^{2}=-M^{2}, M$ being the bound state mass in the 4-point Green's function. The solution of BSE not only yields meson masss, $M$, but also a hadron-quark vertex function that can be used to calculate various meson observables. Now, a number of articles have explored the true nature of QCD's bound-state kernels [10,32-35]. Progress has been made; symmetry preserving Ansatz exist; but the answers are incomplete. But, we do not have the closed form expression for the BSE kernel till date, which can be used to evaluate the confining force. An attempt to obtain closed form expression for kernel in BSE for quark-antiquark bound states using Green's function methods in quantum field theory was made by [33].

We wish to mention that, we do not work in QCD's BSE. Instead, our work is based on QCD motivated BSE in ladder approximation, which is only an approximate description, with an effective four-fermion interaction mediated by a gluonic propagator that serves as the kernel of BSE in the lowest order. We can generalize this to any arbitrary interaction, $K$, where $K$ can be said to represent the sum of all irreducible graphs. The precise form of our kernel in Eq. (4), and (6) is taken in analogy with potential models, which includes a confining term along with a onegluon exchange term. Such effective forms of the BS kernel in ladder BSE have recently been used in [16,36-40], and can predict bound states having a purely relativistic origin (as shown recently in [36]). As mentioned above, the BSE is quite general, and provides an effective description of bound quark-antiquark systems through a suitable choice of input kernel for confinement.

Our approach using BSE under ladder approximation with an effective interaction kernel does not represent the complete dynamics of the system in field theory sense, but it has the practical utility of maintaining the link between spectroscopy and transition amplitudes, that is available through its $3 \mathrm{D}$ reduction using CIA.

Our starting BSE is 4D in all details, including its kernel. By using CIA ansatz on the BS kernel (which provides it a $3 \mathrm{D}$ support $[9,13]$ by postulating that the pairwise BSE kernel is a function of only $\hat{q}$, so that $\hat{q} . P=0$ ), we reduce the $4 \mathrm{D}$ BSE to its 3D form thereby giving up its original 4D form, and using it as a fresh starting point. The reduction of 4D BSE to 3D Salpeter equation is exact in our framework. And by use of 4D BSE with a $4 \times 4$ spinor structure, we get four such Salpeter equations in Eq. (2), which are again covariant due to their dependence on $\hat{q}$, the transverse component of internal momentum. The covariance of 3D Salpeter equations ensures their validity for a hadron in arbitrary motion. The 3D Salpeter equations are reduced to mass spectral equations in an approximate harmonic oscillator basis.

We wish to mention that in the sense of incorporation of input confining potential, and also for mass spectral studies, our BSE framework makes contact with potential models. However the dependence of input kernel on the running QCD coupling constant, $\alpha_{s}$ through the flavor dependent spring constant, $\omega_{q \bar{q}}^{2}$ gives explicit QCD motivation to the BS kernel, as mentioned in details in Sec. II. And, in the process of reduction of $4 \mathrm{D}$ BSE to its $3 \mathrm{D}$ form under CIA, we identify the $4 \mathrm{D}$ hadron-quark vertex function, $\Gamma(\hat{q})$, expressible as in Eq. (3), which is related to the 4D BS wave function through, $\Psi(P, q)=S_{F}\left(p_{1}\right) \Gamma(\hat{q}) S_{F}\left(-p_{2}\right)$, where $\Gamma$ is sandwiched by two quark propagators, and incorporates all the relevant Dirac structures, and is made use of in evaluation of various transition amplitudes through quark-loop diagrams using the techniques of field theory. Thus the practical advantage of using an effective form of interaction kernel in BSE as an input, and using 3D reduction through CIA is mainly due to the ease with which it is able to keep a link between mass spectroscopy and transition amplitudes.

In our works, we are not only interested in studying the mass spectrum of hadrons, which no doubt is an important element to study dynamics of hadrons, but also the hadronic wave functions that play an important role in the calculation of decay constants, form factors, structure functions etc. for $Q \bar{Q}$, and $Q \bar{q}$ hadrons. These hadronic Bethe-Salpeter wave functions calculated algebraically in this work can provide information about the long distance nonperturbative physics, as will be discussed in Sec. V, and Discussions. These wave functions can lead to studies on a number of processes involving $Q \bar{Q}$, and $Q \bar{q}$ states, and provide a guide for experiments. Our basic aim has been to 
develop a model using $4 \times 4$ BSE that can explain both mass spectrum of $Q \bar{Q}$, and $Q \bar{q}$ states as well as their decay widths through various processes using the same set of input parameters that are fixed from their mass spectrum.

In this context, in some of the recent works [41-45], we have been involved in working on the mass spectrum and decay properties of equal mass ground and excited states of scalar, pseudoscalar, vector, and axial vector $Q \bar{Q}$ quarkonia in the framework of a $4 \times 4 \mathrm{BSE}$. These include their leptonic decays, two-photon decays, single photon radiative decays, and two gluon decays of these charmonium $(c \bar{c})$ and bottomonium $(b \bar{b})$ states which have been extensively studied by us in the formulation of BetheSalpeter equation. However, we had not so far generalized this $4 \times 4$ representation for two-body $(Q \bar{Q}) \mathrm{BS}$ amplitude framework to incorporate unequal mass dynamics, which we have now done in the present work. However, the price we have to again pay is to analytically solve a coupled set of equations for all quarkonia, which we have again explicitly shown get decoupled, in spite of the fact that we have used the full structure of the BS wave function, $\psi(\hat{q})$ in calculation of $\gamma_{\mu} \psi(\hat{q}) \gamma_{\mu}$ on the right side of the Salpeter equations. Due to these facts, the system of coupled Salpeter equations encountered in the present work are much more involved and complex than the ones encountered in equal mass quarkonia in [42]. We have explicitly shown that they lead to mass spectral equations with analytical solutions for both masses, as well as eigenfunctions for the ground and excited states for $0^{++}, 0^{-+}$, and $1^{--}$for heavy-light hadrons with quark composition, $c \bar{u}, c \bar{s}, c \bar{b}, b \bar{u}$, and $b \bar{s}$ in an approximate harmonic oscillator basis. We then perturbatively incorporate the one-gluon-exchange (OGE), and solve the spectrum of these states.

We wish to mention that in unequal mass systems such as $Q \bar{q}$, the quarks are not very close together, and the confining interaction dominates over the OGE interactions due to which the perturbative incorporation of OGE term is reasonable approximation. The unequal mass kinematics that also gives the partitioning of internal momenta of the hadron rests on the WightmanGarding definition of internal momenta of individual quarks. The main advantage of our approach in comparison to other BSE approaches is that, we follow analytic methods of solutions for heavy-light quarkonia (whose equations are much more involved than $Q \bar{Q}$ ), that provide a much deeper in sight into the mass spectral problem, and are able to obtain the mass spectrum in terms of the principal quantum number $N$, and also in the process, we get algebraic forms of wave functions that are used for calculations of various transition amplitudes and decay constants of quarkonia, in contrast to the purely numerical approaches followed by the other works [16].
This paper is organized as follows: In Sec. II, we introduce the formulation of the $4 \times 4$ Bethe-Salpeter equation under the covariant instantaneous ansatz, and derive the hadron-quark vertex. In Secs. III, IV, and V, we derive the mass spectral equation of heavy-light scalar, pseudoscalar, and vector mesons respectively. In Secs. VI and VII, we derive the decay constants $f_{P}$ for pseudoscalar, and $f_{V}$ for vector $Q \bar{q}$ states respectively. In Sec. VIII, we provide the numerical results and discussion.

\section{FORMULATION OF THE $4 \times 4$ BETHE-SALPETER EQUATION}

We give here the main points about the $4 \times 4$ BSE under the covariant instantaneous Ansatz (CIA), which is a Lorentz-invariant generalization of instantaneous approximation (IA), which is used to derive the 3D Salpeter equations $[41,42,46]$. We start with a 4D BSE for quarkantiquark system with quarks of constituent masses, $m_{1}$ and $m_{2}$, written in a $4 \times 4$ representation of $4 \mathrm{D}$ BS wave function $\Psi(P, q)$ as:

$S_{F}^{-1}\left(p_{1}\right) \Psi(P, q) S_{F}^{-1}\left(-p_{2}\right)=\frac{i}{(2 \pi)^{4}} \int d^{4} q^{\prime} K\left(q, q^{\prime}\right) \Psi\left(P, q^{\prime}\right)$

where $K\left(q, q^{\prime}\right)$ is the interaction kernel between the quark and antiquark, and $p_{1,2}$ are the momenta of the quark and antiquark, which are related to the internal 4-momentum $q$ and total momentum $P$ of hadron of mass $M$ as, $p_{1,2 \mu}=\hat{m}_{1,2} P_{\mu} \pm q_{\mu}$, where, $\hat{m}_{1,2}=\frac{1}{2}\left[1 \pm \frac{\left(m_{1}^{2}-m_{2}^{2}\right)}{M^{2}}\right]$, always satisfy, $\hat{m}_{1}+\hat{m}_{2}=1$, and is a natural choice that allocates most of the momentum to the heavy quark, while a smaller part of momentum to the lighter quark in a heavy-light meson, but equal momenta to both quarks in $c \bar{c}$ mesons.

Making use of covariant instantaneous Ansatz, where, $K\left(q, q^{\prime}\right)=K\left(\hat{q}, \hat{q}^{\prime}\right)$ on the BS kernel, where $\hat{q}_{\mu}=q_{\mu}-$ $\frac{q . P}{P^{2}} P_{\mu}$ is the component of internal momentum of the hadron that is orthogonal to the total hadron momentum, i.e., $\hat{q} . P=0$, while $\sigma P_{\mu}=\frac{q . P}{P^{2}} P_{\mu}$ is the component of $q$ longitudinal to $P$, where the 4-dimensional volume element is, $d^{4} q=d^{3} \hat{q} M d \sigma$, and following a sequence of steps outlined in [41], we get the covariant forms of four Salpeter equations (in 4D variable $\hat{q}$ ), which are effective 3D forms of BSE, and are valid for hadrons in arbitrary motion. The four independent Salpeter equations are [41]:

$$
\begin{aligned}
\left(M-\omega_{1}-\omega_{2}\right) \psi^{++}(\hat{q}) & =\Lambda_{1}^{+}(\hat{q}) \Gamma(\hat{q}) \Lambda_{2}^{+}(\hat{q}) \\
\left(M+\omega_{1}+\omega_{2}\right) \psi^{--}(\hat{q}) & =-\Lambda_{1}^{-}(\hat{q}) \Gamma(\hat{q}) \Lambda_{2}^{-}(\hat{q}) \\
\psi^{+-}(\hat{q}) & =0 \\
\psi^{-+}(\hat{q}) & =0
\end{aligned}
$$


where $\psi^{ \pm \pm}(\hat{q})=\Lambda_{1}^{ \pm}(\hat{q}) \frac{p}{M} \psi(\hat{q}) \frac{p}{M} \Lambda_{1}^{ \pm}(\hat{q})$, and $\Lambda^{ \pm}$are the projection operators [41] for each of the constituents. Here, $\Gamma(\hat{q})$ is the nonperturbative $4 \mathrm{D}$ hadron-quark vertex function,

$$
\Gamma(\hat{q})=\int \frac{d^{3} \hat{q}^{\prime}}{(2 \pi)^{3}} K\left(\hat{q}, \hat{q}^{\prime}\right) \psi\left(\hat{q}^{\prime}\right),
$$

which enters into the 4D BS wave function, $\Psi(P, q)=$ $S_{F}\left(p_{1}\right) \Gamma(\hat{q}) S_{F}\left(-p_{2}\right)$ (for details, see [42]), which is basically a 3-point function, where the quark, and the anti-quark propagators sandwich the hadron-quark vertex, $\Gamma$ (which is the amputated 3-point function), that is used for calculation of transition amplitudes of various processes. We wish to point out that the 4D BS wave function, $\Psi(P, q)$ is analogous to the quark bilinear 3 -point correlation function used in a recent lattice calculation [47] of nonperturbative structure of vector and axial vector vertices.

We wish to emphasize that the present model after 3D reduction is still covariant. This is due to the fact that we have reduced a fully $4 \mathrm{D}$ BSE to 3D BSE [which are actually four Salpeter equations in Eq. (2)] by use of covariant instantaneous Ansatz (CIA), which is a Lorentzinvariant generalization of the instantaneous approximation (IA). We thus obtain the covariant forms of Salpeter equations, which are effective 3D forms of BSE, and are valid for hadrons in arbitrary motion.

Regarding the interaction kernel $K\left(\hat{q}^{\prime}, \hat{q}\right)$, as mentioned earlier, we use an effective form of interaction kernel, $K$ given below in Eq. (4), and (6), mainly due to the ease with which it is able to keep a link between mass spectroscopy [arising from 3D Salpeter equations in Eq. (2)], and transition amplitudes through a vital connection between the 3D wave function $\psi(\hat{q})$, that satisfies the first two 3D Salpeter equations (that are used for determination of spectra), and the hadron-quark vertex $\Gamma$ (for calculation of transition amplitudes in $4 \mathrm{D}$ basis), as can be seen from the structure of the first two Salpeter equations that connect $\psi(\hat{q})$ [on the left-hand side (LHS)], and $\Gamma(\hat{q})$ [on the right-hand side (RHS)].

The kernel can be written as,

$$
K\left(\hat{q}^{\prime}, \hat{q}\right)=\left(\frac{1}{2} \vec{\lambda}_{1} \cdot \frac{1}{2} \vec{\lambda}_{2}\right)\left(\gamma_{\mu} \otimes \gamma_{\mu}\right) V\left(\hat{q}^{\prime}, \hat{q}\right)
$$

with color, spin, and orbital parts, respectively. For a kernel with the above spin dependence, we can rewrite the hadronquark vertex in Eq. (3) as [42],

$$
\Gamma(\hat{q})=\int \frac{d^{3} \hat{q}^{\prime}}{(2 \pi)^{3}} V\left(\hat{q}, \hat{q}^{\prime}\right) \gamma_{\mu} \psi\left(\hat{q}^{\prime}\right) \gamma_{\mu},
$$

where, each of the $\gamma_{\mu}$ s sandwich the BS wave function, $\psi(\hat{q})$, with the scalar part of the kernel, $V=V_{\mathrm{OGE}}+$ $V_{\text {Confinement }}$ as,

$$
\begin{aligned}
V\left(\hat{q}, \hat{q}^{\prime}\right) & =\frac{4 \pi \alpha_{s}}{\left(\hat{q}-\hat{q}^{\prime}\right)^{2}}+\frac{3}{4} \omega_{q \bar{q}}^{2} \int d^{3} r\left(\kappa r^{2}-\frac{C_{0}}{\omega_{0}^{2}}\right) e^{i\left(\hat{q}-\hat{q}^{\prime}\right) \cdot \vec{r}}, \\
\kappa & =\left(1+4 \hat{m}_{1} \hat{m}_{2} A_{0} M^{2} r^{2}\right)^{-\frac{1}{2}} .
\end{aligned}
$$

To give an idea about the form of confining potential, $V_{\text {conf }}(r)=\frac{3}{4} \omega_{q \bar{q}}^{2}\left(\kappa r^{2}-\frac{C_{0}}{\omega_{0}^{2}}\right)$, in Fig. 1, we have plotted it versus $r$ for the ground (1S) states of $\eta_{b}, B_{c}, B, \eta_{c}$, and $D$, since we are using it only for $Q \bar{Q}$, and $Q \bar{q}$ systems in this work as an illustration of its behavior with the hadron mass. Here $\omega_{q \bar{q}}^{2}=4 \hat{m}_{1} \hat{m}_{2} M^{2} \alpha_{s}\left(M^{2}\right)$ is the flavor dependent spring constant, while $C_{0} / \omega_{0}^{2}$ plays the role of ground state energy. The presence of running coupling constant, $\alpha_{s}$ in $\omega_{q \bar{q}}^{2}$ provides an explicit QCD motivation to the BSE kernel. It is to be seen that this algebraic form of the potential ensures a smooth transition from nearly harmonic (for $c \bar{u}$ ) to almost linear (for $b \bar{b}$ ). It is this algebraic form of the confining potential for which we can see that analytic forms of wave functions can be worked out by solving the 3D Salpeter equations as will be shown later in this paper.

The confinement part with a sequence of steps can be expressed as [41] $V_{c}\left(\hat{q}, \hat{q}^{\prime}\right)=-\frac{3}{4}(2 \pi)^{3} \bar{V}_{c}(\hat{q}) \delta^{3}\left(\hat{q}-\hat{q}^{\prime}\right)$, with $\bar{V}_{c}(\hat{q})=\omega_{q \bar{q}}^{2}\left(\kappa \vec{\nabla}_{\hat{q}}^{2}+\frac{C_{0}}{\omega_{0}^{2}}\right)$, and $\kappa=\left(1-4 \hat{m}_{1} \hat{m}_{2} A_{0} M^{2} \vec{\nabla}_{\hat{q}}^{2}\right)^{-\frac{1}{2}}$.

The present work is a substantial improvement over our previous works $[41,42]$, in the sense that we have taken the full Dirac structure of the 3D BS wave function, $\psi(\hat{q})$ given in Eq. (7) to calculate the spin part, $\gamma_{\mu} \psi(\hat{q}) \gamma_{\mu}$ that enters into the hadron-quark vertex function, $\Gamma(\hat{q})$ as well as the right-hand sides of the 3D coupled integral equations, in contrast to [41,42], where

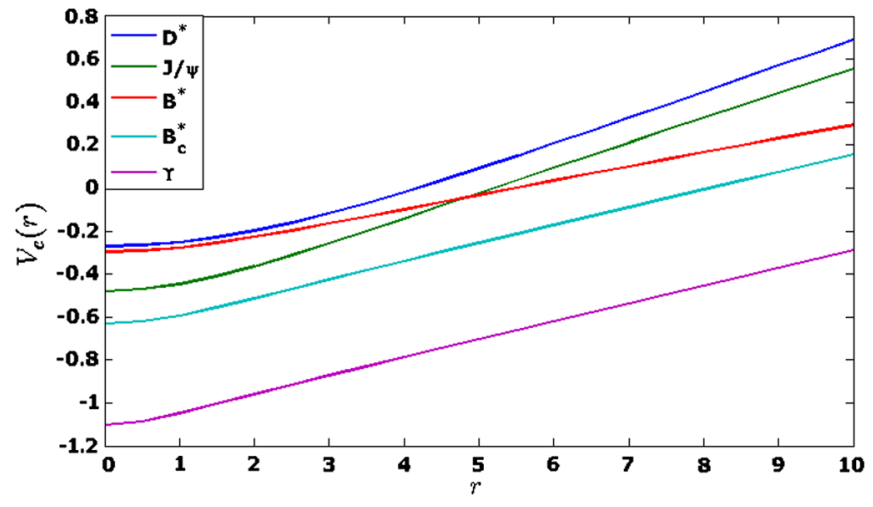

FIG. 1. Plots showing the general behavior of confinement potential, $V_{\text {conf }}(r)=\frac{3}{4} \omega_{q \bar{q}}^{2}\left(\kappa r^{2}-\frac{C_{0}}{\omega_{0}^{2}}\right)$ with $r$ for the ground $(1 S)$ states of $\Upsilon, B_{c}^{*}, B^{*}, J / \Psi$, and $D^{*}$ mesons 
we took only the leading Dirac structures in $\psi(\hat{q})$ to evaluate $\gamma_{\mu} \psi(\hat{q}) \gamma_{\mu}$, in the integrals on the right of the Salpeter equations in $[41,42]$. What we further find is that the higher order terms of $\bar{V}_{c}$ that we had ignored in $[41,42]$ due to negligible coefficients, $\omega_{q \bar{q}}^{4}$ associated with these terms, get effectively cancelled out when we take the full Dirac structure of the wave function, $\psi(\hat{q})$. Also it is seen that the terms $O\left(\hat{q}^{2} / m^{2}\right)$ that entered into the Salpeter equations, and were subsequently ignored in [42] under heavy-quark approximation, get effectively cancelled out in the present calculation of the mass spectral equation with the use of all the Dirac structures. The framework is quite general so far. Thus, to obtain the mass spectral equation, we have to start with the above four Salpeter equations in Eq. (2).

\section{MASS SPECTRAL EQUATION FOR HEAVY-LIGHT SCALAR $0^{++}$QUARKONIA}

We start with the general form of 4D BS wave function for scalar meson $\left(0^{++}\right)$in [48]. Then, making use of the 3D reduction and making use of the fact that $\hat{q} . P=0$, we can write the general decomposition of the instantaneous BS wave function for scalar mesons $\left(J^{p c}=0^{++}\right)$, of dimensionality $M$ being composed of various Dirac structures that are multiplied with scalar functions $f_{i}(\hat{q})$ and various powers of the meson mass $M$ as [42]

$\psi^{S}(\hat{q})=M f_{1}(\hat{q})-i \not P f_{2}(\hat{q})-i \hat{q} f_{3}(\hat{q})-\frac{2 \not \hat{\phi}}{M} f_{4}(\hat{q})$,

Till now these amplitudes $f_{1}$, and $f_{4}$ in the equation above are all independent, and as per the power counting rule $[13,14]$ proposed by us earlier, the $f_{1}$ and $f_{2}$ are the amplitudes associated with the leading Dirac structures, namely $M$ and $\not P$, while $f_{3}$ and $f_{4}$ will be the amplitudes associated with the subleading Dirac structures, namely, $\hat{\phi}$, and $\frac{2 \not P \hat{q}}{M}$.

We now use the last two Salpeter equations $\psi^{+-}(\hat{q})=$ $\psi^{-+}(\hat{q})=0$ in Eq. (2), that can be used to obtain the constraint relations between the scalar functions for unequal mass mesons as given in Eq. (A1) of the Appendix. We wish to mention that due to the two constraint equations, the scalar functions $f_{i}(\hat{q})(i=1, \ldots, 4)$ are no longer all independent, but are tied together by the relations in Eq. (A1) in the Appendix, due to which the amplitudes get mixed up [42].

The first two Salpeter equations of Eq. (2) lead to a set of coupled integral equations, where the full structure of the wave function $\psi^{S}(\hat{q})$ in (A2) is used to evaluate $\gamma_{\mu} \psi^{S}(\hat{q}) \gamma_{\mu}$ on the right-hand sides of these equations. We proceed in the same way as [42], where on the right side of these equations, we first work with the confining interaction, $V_{c}(\hat{q})$. We show that these equations can be decoupled, and reduced to algebraic equations in an approximate harmonic oscillator basis, and solve them analytically. These equations, given as Eq. (A3) in the Appendix are much more involved than the equal mass case [42]. The coupled integral equations and their detailed procedure for reduction to two identical decoupled algebraic equations in amplitudes $f_{1}$ and $f_{2}$ is relegated to the Appendix. The two decoupled algebraic equations obtained this way are

$$
\begin{gathered}
{\left[\frac{M^{2}}{4}-\frac{1}{4}\left(m_{1}+m_{2}\right)^{2}-\hat{q}^{2}\right] f_{3}(\hat{q})} \\
=-\frac{1}{2}\left(m_{1}+m_{2}\right) \bar{V}_{c}(\hat{q}) f_{3}(\hat{q}) \\
{\left[\frac{M^{2}}{4}-\frac{1}{4}\left(m_{1}+m_{2}\right)^{2}-\hat{q}^{2}\right] f_{4}(\hat{q})} \\
=-\frac{1}{2}\left(m_{1}+m_{2}\right) \bar{V}_{c}(\hat{q}) f_{4}(\hat{q}) .
\end{gathered}
$$

It is to be seen here that on the RHS of the above two equations in Eq. (8), we get only the terms that are linear in $\bar{V}_{c}$, (unlike $[41,42]$, where we also obtained quadratic terms of the type, $\bar{V}_{c}^{2}$, that were very small in magnitude in comparison to $\bar{V}_{c}$ ). Since the two equations are of the same form in scalar functions $f_{3}(\hat{q})$ and $f_{4}(\hat{q})$, that are the solutions of identical equations, we can take, $f_{3}(\hat{q}) \approx f_{4}(\hat{q})\left(=\phi_{S}(\hat{q})\right)$. Using the expression for $\bar{V}_{c}(\hat{q})$ given above, we get the equation,

$$
E_{S} \phi_{S}(\hat{q})=\left[-\beta_{S}^{4} \vec{\nabla}_{\hat{q}}^{2}+\hat{q}^{2}\right] \phi_{S}(\hat{q})
$$

where the inverse range parameter $\beta_{S}$ can be expressed as,

$$
\begin{aligned}
\beta_{S} & =\left(\frac{\frac{1}{2} \omega_{q \bar{q}}^{2}\left(m_{1}+m_{2}\right)}{\sqrt{1+8 \hat{m}_{1} \hat{m}_{2} A_{0}\left(N+\frac{3}{2}\right)}}\right)^{1 / 4}, \\
\omega_{q \bar{q}} & =\left(4 M \hat{m}_{1} \hat{m}_{2} \omega_{0}^{2} \alpha_{s}(M)\right)^{1 / 2}, \\
\alpha_{s} & =\frac{12 \pi}{33-2 N_{f}} \log \left(\frac{M^{2}}{\Lambda_{\mathrm{QCD}}^{2}}\right)^{-1}
\end{aligned}
$$

Using the method of power series, this leads to the mass spectral equation for scalar mesons as,

$$
\begin{gathered}
\frac{1}{4}\left[M^{2}-\left(m_{1}+m_{2}\right)^{2}\right]+\frac{C_{0} \beta_{S}^{4}}{\omega_{0}^{2}} \sqrt{1+8 \hat{m}_{1} \hat{m}_{2} A_{0}\left(N+\frac{3}{2}\right)} \\
=2 \beta_{S}^{2}\left(N+\frac{3}{2}\right), \quad N=1,3,5, \ldots,
\end{gathered}
$$

with the energy eigenvalue of the scalar mesons, $E_{S}=2 \beta_{S}^{2}\left(N+\frac{3}{2}\right)$, where $N=2 n+l$, with the principal 
quantum number taking values $n=0,1,2, \ldots$, and the orbital quantum number $l=1$ that corresponds to $P$ wave states, and the solutions of Eq. (9) using the power series method are given by the following normalized wave functions that are similar to the wave functions in [42], except for the inverse range parameter $\beta$ expression that is different from [42] due to the exact treatment of the spin part of the kernel, and also the unequal mass kinematics. They are

$$
\begin{aligned}
\phi_{S}(1 P, \hat{q})= & \sqrt{\frac{2}{3}} \frac{1}{\pi^{3 / 4}} \frac{1}{\beta_{S}^{5 / 2}} \hat{q} e^{-\frac{\hat{q}^{2}}{2 \beta_{S}^{2}}} \\
\phi_{S}(2 P, \hat{q})= & \sqrt{\frac{5}{3}} \frac{1}{\pi^{3 / 4}} \frac{1}{\beta_{S}^{5 / 2}} \hat{q}\left(1-\frac{2 \hat{q}^{2}}{5 \beta_{S}^{2}}\right) e^{-\frac{\hat{q}^{2}}{2 \beta_{S}^{2}}} \\
\phi_{S}(3 P, \hat{q})= & \sqrt{\frac{35}{12}} \frac{1}{\pi^{3 / 4}} \frac{1}{\beta_{S}^{5 / 2}} \hat{q}\left(1-\frac{4 \hat{q}^{2}}{5 \beta_{S}^{2}}+\frac{4 \hat{q}^{4}}{35 \beta_{S}^{4}}\right) e^{-\frac{\hat{q}^{2}}{2 \beta_{S}^{2}}} \\
\phi_{S}(4 P, \hat{q})= & \sqrt{\frac{35}{8}} \frac{1}{\pi^{3 / 4}} \frac{1}{\beta_{S}^{5 / 2}} \hat{q}\left(1-\frac{6 \hat{q}^{2}}{5 \beta_{S}^{2}}+\frac{12 \hat{q}^{4}}{35 \beta_{S}^{4}}-\frac{8 \hat{q}^{6}}{315 \beta_{S}^{6}}\right) \\
& \times e^{-\frac{\hat{q}^{2}}{2 \beta_{S}^{2}}},
\end{aligned}
$$

Now, we treat the mass spectral equation in Eq. (9), which is obtained by taking only the confinement part of the kernel, as an unperturbed spectral equation with the unperturbed wave functions in Eq. (12). We then incorporate the one gluon exchange term in the interaction kernel perturbatively (as in [42]) and solve to first order in perturbation theory. The complete mass spectra of ground and excited states of heavy-light scalar mesons is

$$
\begin{gathered}
\frac{1}{8 \beta_{S}^{2}}\left[M^{2}-\left(m_{1}+m_{2}\right)^{2}\right]+\frac{C_{0} \beta_{S}^{2}}{2 \omega_{0}^{2}} \sqrt{1+8 \hat{m}_{1} \hat{m}_{2} A_{0}\left(N+\frac{3}{2}\right)} \\
+\gamma\left\langle V_{\text {coul }}^{S}\right\rangle=N+\frac{3}{2}, \quad N=1,3,5, \ldots
\end{gathered}
$$

where $\left\langle V_{\text {coul }}^{S}\right\rangle$ is the expectation value of $V_{\text {coul }}^{S}$ between the unperturbed states of the scalar mesons with $l=1$ and $n=0,1,2, \ldots$, and $\gamma$ is introduced as a weighting factor to have the Coulomb term dimensionally consistent with the harmonic term, with $\gamma$ is expressed in units of $\omega_{0}^{4} /\left(C_{0} \beta^{2}\right)$, and it also acts as a measure of the strength of the perturbation. The expectation value of the Coulomb term associated with the OGE term for scalar quarkonia is a single elegant expression for all states, $|n P\rangle$, (where, $n=1,2,3, \ldots)$,

$$
\left\langle n P\left|V_{\text {coul }}^{S}\right| n P\right\rangle=-\frac{32 \pi \alpha_{s}}{9 \beta_{S}^{2}} .
$$

The results of our model for mass spectrum for scalar $Q \bar{q}$ states along with data [21], and other models is given in
Table II. It is observed that the mass spectra of mesons of various $J^{P C}\left(0^{++}, 0^{-+}\right.$, and $\left.1^{--}\right)$is somewhat insensitive to a small range of variations of parameter $\omega_{0}$, as long as $\frac{C_{0}}{\omega_{0}^{2}}$ is a constant. The input parameters of our model obtained by best fit to the spectra of ground states of scalar, pseudoscalar, and vector $Q \bar{q}$, and $Q \bar{Q}$ quarkonia are $C_{0}=0.69$, $\omega_{0}=0.22 \mathrm{GeV}, \Lambda_{\mathrm{QCD}}=0.250 \mathrm{GeV}$, and $A_{0}=0.01$, with input quark masses $m_{u}=0.300 \mathrm{GeV}, m_{s}=0.430 \mathrm{GeV}$, $m_{c}=1.490 \mathrm{GeV}$, and $m_{b}=4.690 \mathrm{GeV}$. Using these set of input parameters, we do the mass spectral calculations of both ground and excited states of heavy-light scalar $\left(0^{++}\right)$ (in Sec. III), pseudoscalar $\left(0^{-+}\right)$(in Sec. IV), and vector $\left(1^{--}\right)$(in Sec. V) quarkonia.

Further, we have found numerical values of perturbation strength, $\gamma$ multiplying $V_{\text {coulomb }}$ that gave reasonable agreement with data and other models is difficult to be expressed in terms of a single algebraic form with dimension, $M^{2}$ - that is required to have $V_{\text {coulomb }}$ to be dimensionally consistent with $V_{\text {confinemt }}$ in the mass spectral equations, when we want to study both ground and excited states of all possible $Q \bar{q}$ mesons, though we can obtain this algebraic form of $\gamma$ for equal quark mesons ( $c \bar{c}$ states) as in [42]. Hence we label these values of $\gamma$ in Table I, which can at best be expressed in multiples of $\omega_{0}^{4} /\left(C_{0} \beta^{2}\right)$, where it is to be noted that $\beta$ is an effective range parameter given by Eq. (10), and is not an input parameter of the model. The values of $\gamma$ for various mesons is given in the Table I.

We also calculated percentage contribution of the Coulomb term to the mass of each meson state, which are indeed small, as seen in Table I, justifying the perturbative treatment of the Coulomb term for these states. We see that for any $J^{P C}$, the contribution of Coulomb term to meson mass for $b \bar{u}, b \bar{s}$, and $c \bar{b}$ mesons is larger than the corresponding contributions from $c \bar{u}, c \bar{s}$, and $c \bar{c}$ states. Also, as we go to higher radial states of a given meson, the contribution of Coulomb term to mass keeps decreasing from its corresponding contribution for ground states. This means that the radially excited states are loosely bound in comparison to the ground states, which is similar to the case of atoms.

We now derive the mass spectral equations of unequal mass pseudoscalar mesons in the next section.

TABLE I. Values of strength of perturbation $\gamma$ in units of $\mathrm{GeV}^{2}$.

\begin{tabular}{ll}
\hline \hline Mesons & \multicolumn{1}{c}{$\gamma$} \\
\hline$\eta_{c}(n S), \Psi(n S)$ & 0.01 \\
$\chi_{c 0}(n P)$ & 0.06 \\
$B(n S), B_{s}(n S), B^{*}(n S), B_{s}^{*}(n S)$ & 0.05 \\
$B_{c}(n S), B_{c}^{*}(n S)$ & 0.09 \\
$D(n S), D_{s}(n S), D^{*}(n S), D_{s}^{*}(n S)$ & 0.002 \\
$D(n P), D_{s}(n P)$ & 0.02 \\
\hline \hline
\end{tabular}


TABLE II. Masss spectra of ground and excited states of scalar $0^{++}$quarkonia (in GeV) in BSE-CIA (with the percentage contribution of the OGE to meson mass) along with data and results of other models.

\begin{tabular}{|c|c|c|c|c|c|c|c|}
\hline & BSE-CIA & $\%$ contribution of OGE & Expt. [21] & BSE-SDE & $\mathrm{PM}$ & Lattice QCD & RQM \\
\hline$M_{B_{c}\left(1 P_{0}\right)}$ & 6.7183 & $11.23 \%$ & & $6.490[35]$ & $6.715[49]$ & $6.727 \pm 0.03[50]$ & $6.699[51]$ \\
\hline$M_{B_{c}\left(2 P_{0}\right)}$ & 7.1788 & $9.55 \%$ & & & $7.102[49]$ & & $7.091[51]$ \\
\hline$M_{B_{c}\left(3 P_{0}\right)}$ & 7.6477 & $8.20 \%$ & & & & & \\
\hline$M_{B_{c}\left(4 P_{0}\right)}$ & 8.1157 & $7.12 \%$ & & & & & \\
\hline$M_{B_{c}\left(5 P_{0}\right)}$ & 8.5772 & $6.24 \%$ & & & & & \\
\hline$M_{B_{s}\left(1 P_{0}\right)}$ & 5.8774 & $12.53 \%$ & & $5.701[35]$ & $5.812[52]$ & & $5.833[53]$ \\
\hline$M_{B_{s}\left(2 P_{0}\right)}$ & 6.2827 & $10.63 \%$ & & & $6.367[52]$ & & 6.318 [53] \\
\hline$M_{B_{s}\left(3 P_{0}\right)}$ & 6.7218 & $9.02 \%$ & & & $6.879[52]$ & & \\
\hline$M_{B_{s}\left(4 P_{0}\right)}$ & 7.1742 & $7.71 \%$ & & & & & \\
\hline$M_{B_{s}\left(5 P_{0}\right)}$ & 7.6270 & $6.66 \%$ & & & & & \\
\hline$M_{B\left(1 P_{0}\right)}$ & 5.7386 & $12.08 \%$ & & $5.610[35]$ & $5.730[52]$ & & $5.749[53]$ \\
\hline$M_{B\left(2 P_{0}\right)}$ & 6.1349 & $10.25 \%$ & & & $6.297[52]$ & & $6.221[53]$ \\
\hline$M_{B\left(3 P_{0}\right)}$ & 6.5709 & $8.67 \%$ & & & $6.826[52]$ & & \\
\hline$M_{B\left(4 P_{0}\right)}$ & 7.0231 & $7.39 \%$ & & & & & \\
\hline$M_{B\left(5 P_{0}\right)}$ & 7.4769 & $6.37 \%$ & & & & & \\
\hline$M_{D_{s}\left(1 P_{0}\right)}$ & 2.3873 & $2.65 \%$ & $2.3177 \pm 0.0006$ & & $2.4945[54]$ & & $2.509[53]$ \\
\hline$M_{D_{s}\left(2 P_{0}\right)}$ & 2.9531 & $1.57 \%$ & & & $3.0004[54]$ & & $3.054[53]$ \\
\hline$M_{D_{s}\left(3 P_{0}\right)}$ & 3.4757 & $1.06 \%$ & & & & & \\
\hline$M_{D_{s}\left(4 P_{0}\right)}$ & 3.9558 & $0.78 \%$ & & & & & \\
\hline$M_{D\left(1 P_{0}\right)}$ & 2.3500 & $5.60 \%$ & $2.318 \pm 0.029$ & $2.300[35]$ & $2.3864[54]$ & & $2.406[53]$ \\
\hline$M_{D\left(2 P_{0}\right)}$ & 2.8983 & $3.32 \%$ & & & $2.8884[54]$ & & $2.919[53]$ \\
\hline$M_{D\left(3 P_{0}\right)}$ & 3.4082 & $2.24 \%$ & & & & & \\
\hline$M_{D\left(4 P_{0}\right)}$ & 3.8776 & $1.64 \%$ & & & & & \\
\hline$M_{\chi_{c 0}\left(1 P_{0}\right)}$ & 3.4122 & $6.87 \%$ & $3.4147 \pm 0.00030$ & & $3.440[4]$ & & 3.413 [18] \\
\hline$M_{\chi_{c 0}\left(2 P_{0}\right)}$ & 3.9667 & $4.76 \%$ & $3.918 \pm 0.0019$ & $3.8368[16]$ & $3.920[4]$ & & $3.870[18]$ \\
\hline$M_{\chi_{c 0}\left(3 P_{0}\right)}$ & 4.4858 & $3.54 \%$ & & & & $4.301[18]$ & \\
\hline$M_{\chi_{c 0}\left(4 P_{0}\right)}$ & 4.9737 & $2.77 \%$ & & & & & \\
\hline$M_{\chi_{c 0}\left(5 P_{0}\right)}$ & 5.4343 & $2.24 \%$ & & & & & \\
\hline
\end{tabular}

IV. MASS SPECTRAL EQUATIONS FOR HEAVYLIGHT PSEUDOSCALAR $0^{-+}$QUARKONIA

The general decomposition for the 3D wave function of pseudoscalar mesons obtained from the general 4D form [48] through 3D reduction as in previous section can be written as [42]

$\psi^{P}(\hat{q})=\left[M \phi_{1}(\hat{q})-i \not P \phi_{2}(\hat{q})+i \hat{\not} \phi \phi_{3}(\hat{q})+\frac{\not P}{M} \phi_{4}(\hat{q})\right] \gamma_{5}$

We use the last two Salpeter equations in Eq. (2) to find the equations of constraints on the components of the wave function as given in Eq. (A4) of the Appendix, that relate the amplitudes, $\phi_{4}$ with $\phi_{2}$, and $\phi_{3}$ with $\phi_{1}$, and hence causing a mixing up of the amplitudes as in the scalar meson case.

We then use the first two Salpeter equations of Eq. (2) to obtain the corresponding coupled integral equations of pseudoscalar mesons (with use of confining interaction alone) as in Eq. (A6) of Appendix. Using the same procedure as in the case of scalar mesons, these two equations can be decoupled, and reduced to two independent algebraic equations:

$$
\begin{gathered}
{\left[\frac{M^{2}}{4}-\frac{1}{4}\left(m_{1}+m_{2}\right)^{2}-\hat{q}^{2}\right] \phi_{1}(\hat{q})} \\
=-\frac{1}{2}\left(m_{1}+m_{2}\right) \bar{V}_{c}(\hat{q}) \phi_{1}(\hat{q}) \\
{\left[\frac{M^{2}}{4}-\frac{1}{4}\left(m_{1}+m_{2}\right)^{2}-\hat{q}^{2}\right] \phi_{2}(\hat{q})} \\
=-\frac{1}{2}\left(m_{1}+m_{2}\right) \bar{V}_{c}(\hat{q}) \phi_{2}(\hat{q}) .
\end{gathered}
$$

Here, we again see that the scalar functions $\phi_{1}(\hat{q})$ and $\phi_{2}(\hat{q})$ satisfy identical equations, and can be taken as $\phi_{1}(\hat{q}) \approx \phi_{2}(\hat{q})\left(=\phi_{P}(\hat{q})\right)$. Using the expression for $\bar{V}_{c}(\hat{q})$ after Eq. (6), we obtain the mass spectral equation as,

$$
E_{P} \phi_{P}(\hat{q})=\left[-\beta_{P}^{4} \vec{\nabla}_{\hat{q}}^{2}+\hat{q}^{2}\right] \phi_{P}(\hat{q}),
$$

whose solutions give the unperturbed mass spectrum (due to confining interactions alone), 


$$
\begin{gathered}
\frac{1}{8}\left[M^{2}-\left(m_{1}+m_{2}\right)^{2}\right]+\frac{C_{0} \beta_{P}^{4}}{2 \omega_{0}^{2}} \sqrt{1+8 \hat{m}_{1} \hat{m}_{2} A_{0}\left(N+\frac{3}{2}\right)} \\
=\left(N+\frac{3}{2}\right) \beta_{P}^{2} ; \quad N=2 n+l
\end{gathered}
$$

with the orbital quantum number $l=0$ that corresponds to the $S$ states, and $\beta_{P}=\left(\frac{\frac{1}{2} \omega_{q \bar{q}}^{2}\left(m_{1}+m_{2}\right)}{\sqrt{1+8 \hat{m}_{1} \hat{m}_{2} A_{0}\left(N+\frac{3}{2}\right)}}\right)^{1 / 4}$. This unperturbed mass spectral equation of pseudoscalar meson is the same as the corresponding spectral equation of scalar meson in Eq. (9), except that $\beta_{S}$ is replaced by $\beta_{P}$, and $\phi_{S}(\hat{q})$ replaced by $\phi_{P}(\hat{q})$. The normalized unperturbed wave functions of $1 S, \ldots, 4 S$ states of pseudoscalar meson with $l=0$ derived analytically using the power series method of solution of Eq. (17) are

$\phi_{P}(1 S, \hat{q})=\frac{1}{\pi^{3 / 4}} \frac{1}{\beta_{P}^{3 / 2}} e^{-\frac{\hat{q}^{2}}{2 \beta_{P}^{2}}}$

$\phi_{P}(2 S, \hat{q})=\sqrt{\frac{3}{2}} \frac{1}{\pi^{3 / 4}} \frac{1}{\beta_{P}^{3 / 2}}\left(1-\frac{2 \hat{q}^{2}}{3 \beta_{P}^{2}}\right) e^{-\frac{\hat{q}^{2}}{2 \beta_{P}^{2}}}$

$\phi_{P}(3 S, \hat{q})=\sqrt{\frac{15}{8}} \frac{1}{\pi^{3 / 4}} \frac{1}{\beta_{P}^{3 / 2}}\left(1-\frac{4 \hat{q}^{2}}{3 \beta_{P}^{2}}+\frac{4 \hat{q}^{4}}{15 \beta_{P}^{4}}\right) e^{-\frac{\hat{q}^{2}}{2 \beta_{P}^{2}}}$

$\phi_{P}(4 S, \hat{q})=\sqrt{\frac{35}{16}} \frac{1}{\pi^{3 / 4}} \frac{1}{\beta_{P}^{3 / 2}}\left(1-\frac{2 \hat{q}^{2}}{\beta_{P}^{2}}+\frac{4 \hat{q}^{4}}{5 \beta_{P}^{4}}-\frac{8 \hat{q}^{6}}{105 \beta_{P}^{6}}\right) e^{-\frac{\hat{q}^{2}}{2 \beta_{P}^{2}}}$

We again incorporate the Coulomb term $V_{\text {coul }}^{P}$ associated with the one gluon exchange interaction perturbatively into the original mass spectral equation of pseudoscalar mesons, giving us the complete mass spectra of ground and excited states of heavy-light pseudoscalar quarkonia with orbital quantum number $l=0$ as

$$
\begin{gathered}
\frac{1}{8 \beta_{P}^{2}}\left[M^{2}-\left(m_{1}+m_{2}\right)^{2}\right]+\frac{C_{0} \beta_{P}^{2}}{2 \omega_{0}^{2}} \sqrt{1+8 \hat{m}_{1} \hat{m}_{2} A_{0}\left(N+\frac{3}{2}\right)} \\
+\gamma\left\langle V_{\text {coul }}^{P}\right\rangle=N+\frac{3}{2}, \quad N=0,2,4, \ldots,
\end{gathered}
$$

where again the perturbation strength $\gamma$ has the same form as in the case of scalar mesons, while, the first order correction to the total energy of the system $E_{P}$ is given by the expectation value of the Coulomb term between the unperturbed states of pseudoscalar mesons $\phi_{P}(n S, \hat{q})$ as

$$
\left\langle n S\left|V_{\text {coul }}^{P}\right| n S\right\rangle=-\frac{32 \pi \alpha_{s}}{3 \beta_{P}^{2}} .
$$

The results of our model for pseudoscalar $Q \bar{q}$ mesons along with data [21] and other models is given in Table III.

We now give the derivation of the mass spectral equations of vector mesons in the next section.

\section{MASS SPECTRAL EQUATIONS FOR HEAVY-LIGHT VECTOR $1^{--}$ QUARKONIA}

We again start with the general 4D decomposition [48]. Using 3D decomposition, the wave function of vector mesons can be written as $[41,42]$ :

$$
\begin{aligned}
\psi^{V}(\hat{q})= & i M \phi \chi_{1}(\hat{q})+\phi \not P \chi_{2}(\hat{q})+[\phi \hat{\phi}-\hat{q} \cdot \epsilon] \chi_{3}(\hat{q}) \\
& -i[P \phi \dot{\phi} \hat{q}+\hat{q} \cdot \epsilon \not P] \frac{1}{M} \chi_{4}(\hat{q})+(\hat{q} \cdot \epsilon) \chi_{5}(\hat{q}) \\
& -i \hat{q} \cdot \epsilon \frac{P}{M} \chi_{6}(\hat{q})
\end{aligned}
$$

We first obtain the constraint equations on the components of the wave functions $(\chi \mathrm{s})$ using the last two Salpeter equations of (2) as in Eq. (A7) in the Appendix, where the amplitude, $\chi_{5}$ is expressed in terms of $\chi_{1}$, and $\chi_{4}$ in terms of $\chi_{2}$, while the amplitudes, $\chi_{3}$ and $\chi_{6}$ vanish. Substituting these constraint relations into Eq. (22) gives us the wave function expressible in terms of the amplitudes, $\chi_{1}$, and $\chi_{2}$ as in Eq. (A8) of Appendix.

Using the first two Salpeter equations, we obtain the coupled integral equations of vector mesons (with confining interaction alone) as in Eq. (A9) of Appendix. Now, using the same procedures to decouple these equations as in the case of scalar and pseudoscalar mesons, we obtain two decoupled algebraic equations,

$$
\begin{gathered}
{\left[\frac{M^{2}}{4}-\frac{1}{4}\left(m_{1}+m_{2}\right)^{2}-\hat{q}^{2}\right] \chi_{1}(\hat{q})} \\
=-\frac{1}{2}\left(m_{1}+m_{2}\right) \bar{V}_{c}(\hat{q}) \chi_{1}(\hat{q}) \\
{\left[\frac{M^{2}}{4}-\frac{1}{4}\left(m_{1}+m_{2}\right)^{2}-\hat{q}^{2}\right] \chi_{2}(\hat{q})} \\
=-\frac{1}{2}\left(m_{1}+m_{2}\right) \bar{V}_{c}(\hat{q}) \chi_{2}(\hat{q})
\end{gathered}
$$

Here, we see that the scalar functions $\chi_{1}(\hat{q})$ and $\chi_{2}(\hat{q})$ satisfy identical equations, and can be taken as $\chi_{1}(\hat{q}) \approx$ $\chi_{2}(\hat{q})=\phi_{V}(\hat{q})$. We then obtain a single differential equation, which is nothing but the equation of a simple quantum mechanical $3 D$-harmonic oscillator with coefficients depending on the hadron mass $M$, and total quantum number $N$. The wave function satisfies the 3D BSE:

$$
\begin{aligned}
& {\left[\frac{M^{2}}{4}-\frac{1}{4}\left(m_{1}+m_{2}\right)^{2}+\frac{C_{0} \beta_{V}^{4}}{\kappa \omega_{0}^{2}}\right] \phi_{V}(\hat{q})} \\
& =\left[-\beta_{V}^{4} \vec{\nabla}_{\hat{q}}^{2}+\hat{q}^{2}\right] \phi_{V}(\hat{q}),
\end{aligned}
$$

which can be rewritten as

$$
E_{V} \phi_{V}(\hat{q})=\left[-\beta_{V}^{4} \vec{\nabla}_{\hat{q}}^{2}+\hat{q}^{2}\right] \phi_{V}(\hat{q}),
$$


where $\beta_{V}=\left(\frac{\frac{1}{2} \omega_{q \bar{q}}^{2}\left(m_{1}+m_{2}\right)}{\sqrt{1+8 \hat{m}_{1} \hat{m}_{2} A_{0}\left(N+\frac{3}{2}\right)}}\right)^{1 / 4}$ is the inverse range parameter, and the total energy of the system is identified as

$$
\begin{aligned}
E_{V}= & \frac{1}{4}\left[M^{2}-\left(m_{1}+m_{2}\right)^{2}\right] \\
& +\frac{C_{0} \beta_{V}^{4}}{\omega_{0}^{2}} \sqrt{1+8 \hat{m}_{1} \hat{m}_{2} A_{0}\left(N+\frac{3}{2}\right)} .
\end{aligned}
$$

This mass spectral equation of vector meson is the same as the corresponding equation of scalar meson in Eq. (9), except that $\beta_{S}$ is replaced by $\beta_{V}$, and $\phi_{S}(\hat{q})$ replaced by $\phi_{V}(\hat{q})$. Therefore, the normalized wave functions of $1 \mathrm{~S}, \ldots, 3 \mathrm{D}$ states of vector meson derived analytically using the power series method of solution of Eq. (25), with $\mathrm{S}$ and $\mathrm{D}$ states corresponding to $l=0$ and $l=2$ respectively, are

$$
\begin{aligned}
\phi_{V}(1 S, \hat{q}) & =\frac{1}{\pi^{3 / 4}} \frac{1}{\beta_{V}^{3 / 2}} e^{-\frac{\hat{q}^{2}}{2 \beta_{V}^{2}}} \\
\phi_{V}(2 S, \hat{q}) & =\sqrt{\frac{3}{2}} \frac{1}{\pi^{3 / 4}} \frac{1}{\beta_{V}^{3 / 2}}\left(1-\frac{2 \hat{q}^{2}}{3 \beta_{V}^{2}}\right) e^{-\frac{\hat{q}^{2}}{2 \beta_{V}^{2}}} \\
\phi_{V}(1 D, \hat{q}) & =\sqrt{\frac{4}{15}} \frac{1}{\pi^{3 / 4}} \frac{1}{\beta_{V}^{7 / 2}} \hat{q}^{2} e^{-\frac{\hat{q}^{2}}{2 \beta_{V}^{2}}} \\
\phi_{V}(3 S, \hat{q}) & =\sqrt{\frac{15}{8}} \frac{1}{\pi^{3 / 4}} \frac{1}{\beta_{V}^{3 / 2}}\left(1-\frac{4 \hat{q}^{2}}{3 \beta_{V}^{2}}+\frac{4 \hat{q}^{4}}{15 \beta_{V}^{4}}\right) e^{-\frac{\hat{q}^{2}}{2 \beta_{V}^{2}}} \\
\phi_{V}(2 D, \hat{q})= & \sqrt{\frac{14}{15}} \frac{1}{\pi^{3 / 4}} \frac{1}{\beta_{V}^{7 / 2}} \hat{q}^{2}\left(1-\frac{2 \hat{q}^{2}}{7 \beta_{V}^{2}}\right) e^{-\frac{\hat{q}^{2}}{2 \beta_{V}^{2}}} \\
\phi_{V}(4 S, \hat{q})= & \sqrt{\frac{35}{16}} \frac{1}{\pi^{3 / 4}} \frac{1}{\beta_{V}^{3 / 2}}\left(1-\frac{2 \hat{q}^{2}}{\beta_{V}^{2}}+\frac{4 \hat{q}^{4}}{5 \beta_{V}^{4}}-\frac{8 \hat{q}^{6}}{105 \beta_{V}^{6}}\right) \\
\phi_{V}(3 D, \hat{q})= & \sqrt{\frac{21}{10}} \frac{1}{\pi^{3 / 4}} \frac{1}{\beta_{V}^{7 / 2}} \hat{q}^{2}\left(1-\frac{4 \hat{q}^{2}}{7 \beta_{V}^{2}}+\frac{4 \hat{q}^{4}}{63 \beta_{V}^{4}}\right) e^{-\frac{\hat{q}^{2}}{2 \beta_{V}^{2}}}
\end{aligned}
$$

Equations (25)-(26) would lead to degenerate masses for $S$ and $D$ states of $Q \bar{q}$, and $Q \bar{Q}$ systems. To get $S-D$ mass splitting, we make use of degenerate perturbation theory. The Coulomb term $V_{\text {coul }}^{V}$ associated with the one gluon exchange interaction is perturbatively incorporated into the mass spectral equation, Eq. (25) (that is treated as the unperturbed equation) for vector mesons, as:

$$
E_{V} \phi_{V}(\hat{q})=\left[-\beta_{V}^{4} \vec{\nabla}_{\hat{q}}^{2}+\hat{q}^{2}+V_{\text {coul }}^{V}\right] \phi_{V}(\hat{q}) .
$$

The complete mass spectral equation of heavy-light vector quarkonia can be put as

$$
\begin{gathered}
\frac{1}{8 \beta_{V}^{2}}\left[M^{2}-\left(m_{1}+m_{2}\right)^{2}\right]+\frac{C_{0} \beta_{V}^{2}}{2 \omega_{0}^{2}} \sqrt{1+8 \hat{m}_{1} \hat{m}_{2} A_{0}\left(N+\frac{3}{2}\right)} \\
+\gamma\left\langle V_{\text {coul }}^{V}\right\rangle=N+\frac{3}{2}, \quad N=0,2,4, \ldots,
\end{gathered}
$$

where $\left\langle V_{\text {coul }}^{V}\right\rangle$ is given by the expectation value of the Coulomb term with respect to the unperturbed states of vector mesons, in Eq. (27). In the secular equation, the only nonzero expectation values of $\left\langle V_{\text {coul }}^{V}\right\rangle$ are the ones that connect states of the same quantum numbers, $n$ and $l$. They are

$$
\begin{aligned}
\left\langle n S\left|V_{\text {coul }}^{V}\right| n S\right\rangle & =-\frac{32 \pi \alpha_{s}}{3 \beta_{V}^{2}} \\
\left\langle n D\left|V_{\text {coul }}^{V}\right| n D\right\rangle & =-\frac{32 \pi \alpha_{s}}{15 \beta_{V}^{2}} .
\end{aligned}
$$

We are giving plots of wave functions, $\phi(n P)$, and $\phi(n S)$ for ground and excited states of $0^{++}$, and $0^{-+}$respectively, $[\phi(n S)$ and $\phi(n D)]$ for $1^{--}$in Figs. 2-4, respectively, which are the unperturbed (long range) wave functions.

Regarding the wave functions, we have obtained the general expressions of 3D forms of long distance (nonperturbative) Bethe-Salpeter wave functions in Eqs. (12), (19), and (27) for $0^{++}, 0^{-+}$, and $1^{--}$respectively. We have given the plots of these wave functions as a function of the internal momentum, $\hat{q}$ in Figs. 2-4. For $Q \bar{q}$ systems, the wave functions show a damped oscillatory behavior, with amplitude for all the $n(n=1,2,3, \ldots)$ states (of $0^{-+}$, and $1^{--}$), being maximum at $0 \mathrm{GeV}$ (confinement region), and falling gradually with increase in $\hat{q}$, and finally becoming zero. For $0^{++}$mesons, the amplitude of the wave function is 0 at $|\hat{q}|=0$ (due to the wave functions being odd), then with increase in $\hat{q}$, it reaches a maximum, executes a damped oscillatory behavior, and finally becoming 0 . Further, as regards all the $0^{++}$mesons, we see that $1 P$ states have zero nodes, followed by $2 P$ states with one node and $3 P$ states with two nodes. A similar trend is observed for $(n S)$ states of $0^{-+}$; and $[(n S)$, and $(n D)]$ states of $1^{--}$ mesons. Thus, these plots show that the $3 \mathrm{D}$ wave functions, $\phi(n P), \phi(n S), \phi(n D)$ and have $(n-1)$ nodes, which is a general feature of bound quantum mechanical systems. An interesting feature of these plots is that as the mass of the meson, $M$ increases, $\phi(\hat{q}) \rightarrow 0$ at a higher value of $|\hat{q}|$. As seen from the plots, the wave functions of heavier mass $Q \bar{q}$ systems (such as $B_{c}, B_{c}^{*}, B_{s}, B_{s}^{*}, B, B^{*}$ ) extend to a much shorter distance than the wave functions of $\left(\chi_{c 0}, \eta_{c}, J / \Psi, D, D^{*}, D_{s}, D_{s}^{*}\right)$, implying thereby that the heavier mesons $\left(B_{c}, B_{c}^{*}, B_{s}, \ldots\right)$ are more tightly bound than the comparatively lighter mesons $\left(\chi_{c 0}, \eta_{c}\right.$, $\left.J / \Psi, D, D^{*}, D_{s}, \ldots\right)$. 
This feature is also supported by the fact that in general, the percentage contribution of $V_{\text {coul }}$ to meson mass, $M$, is larger for $B_{c}, B_{c}^{*} B, B^{*}, B_{s}, B_{s}^{*}$ than $\eta_{c}, \chi_{c 0}, D, D^{*}, D_{s}$, and $D_{s}^{*}$ mesons, as seen in the mass spectrum tables, II, III, and IV for $0^{++}, 0^{-+}$, and $1^{--}$respectively. It is in this sense, the algebraic forms of 3D hadronic BS wave functions can not only provide information about the long
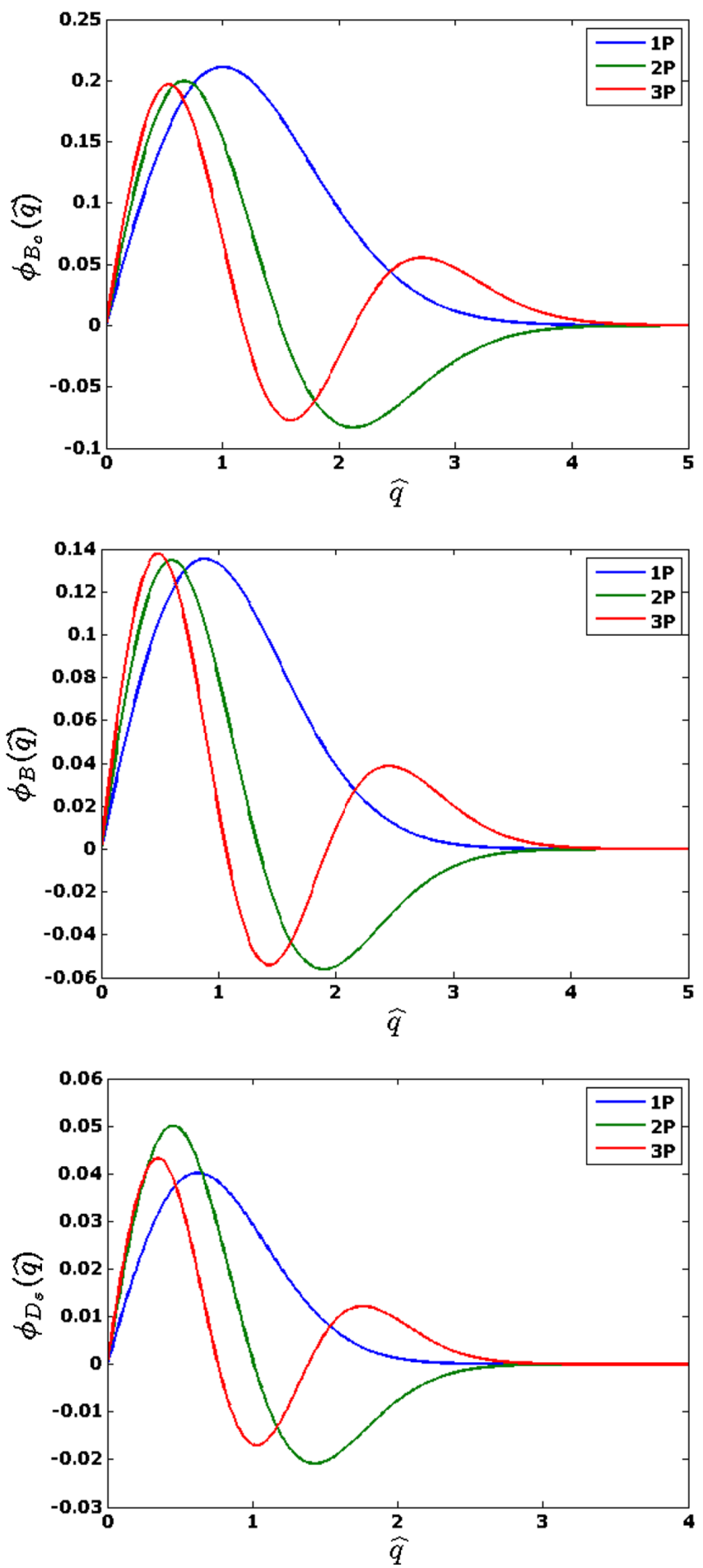

range nonperturbative physics, but also tell us the shortest distance to which they can penetrate to in a hadron. It is in this sense the computed wave functions are physically reasonable and can build a "bridge" between the long distance nonperturbative physics, and the short distance perturbative physics. However as regards mass spectral calculation, we do find some exceptions-for instance,
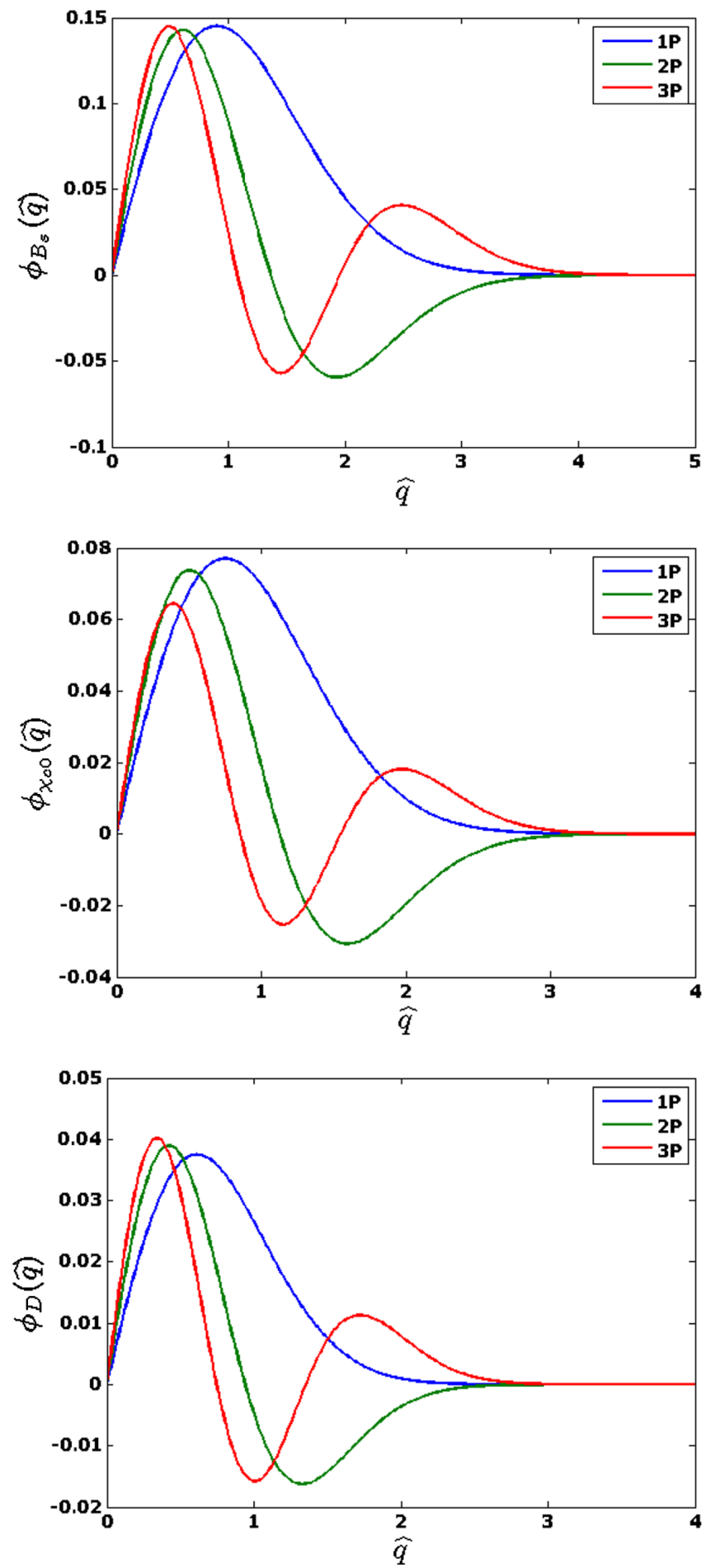

FIG. 2. Plots of wave functions for states $(1 P, \ldots, 3 P) \mathrm{Vs} \hat{q}$ (in Gev.) for scalar mesons, such as; $B_{c}, B_{s}, B, \chi_{c 0}, D_{s}$, and $D$ respectively. 

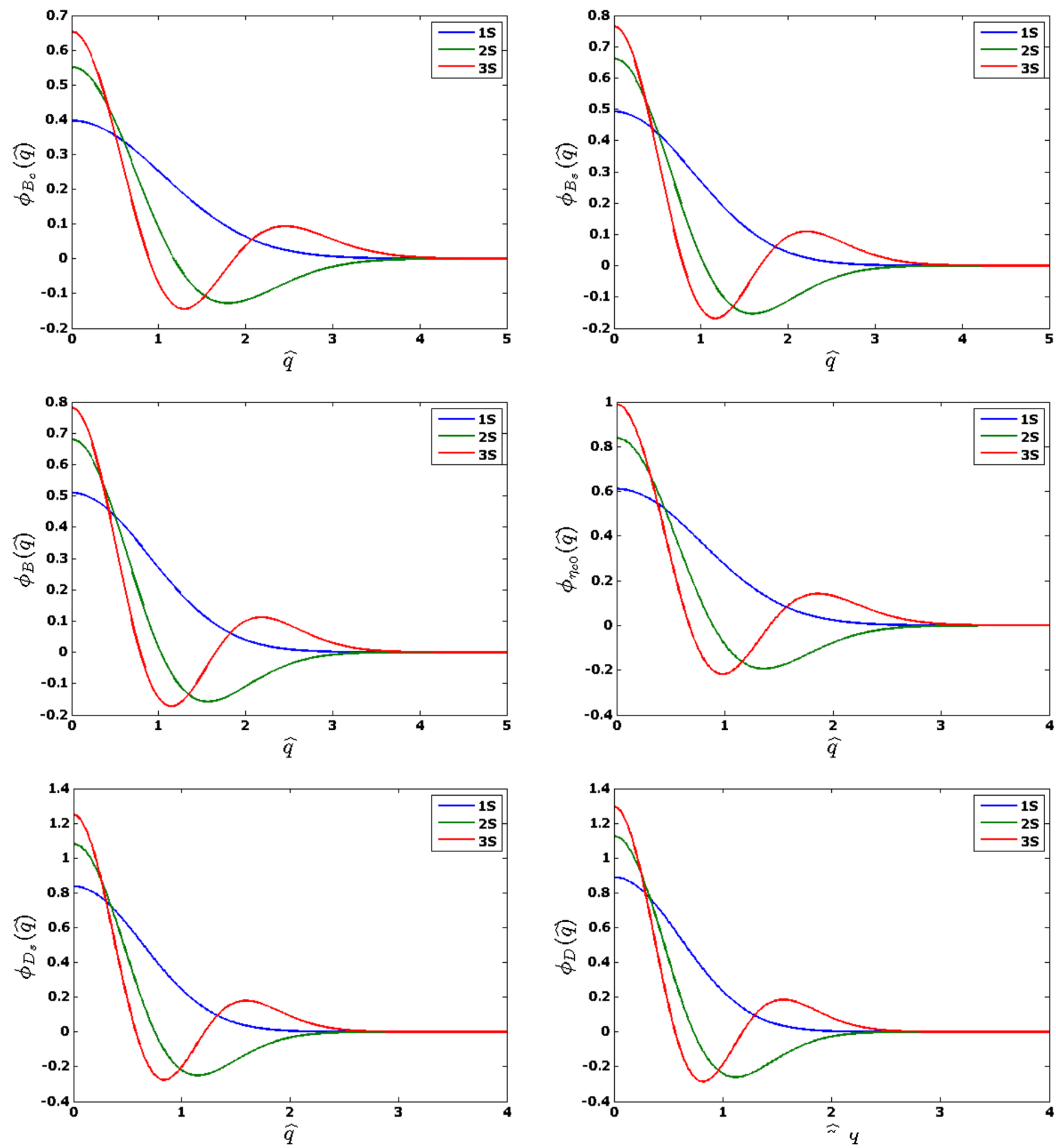

FIG. 3. Plots of wave functions for states $(1 S, \ldots, 3 S) \mathrm{Vs} \hat{q}$ (in Gev.) for pseudoscalar mesons, such as $B_{c}, B_{s}, B, \eta_{c}, D_{s}$, and $D$, respectively.

$J / \Psi(1 S)$ has comparable percentage contribution of $V_{\text {coul }}$ to its mass as the heavier mesons, $B^{*}(1 S)$, and $B_{s}^{*}(1 S)$. This may be argued from the plots of confining potential $V_{\text {conf }}$ in Fig.1, which show that as one moves through $c \bar{u}$, to $c \bar{c}$, the confining potential gets deeper (i.e. more attractive). Similar trend of confining potential is again observed as one goes from $b \bar{u}$ to $b \bar{b}$. This may be a pointer to the fact that in equal mass mesons (such as $c \bar{c}$, and $b \bar{b}$ ), the distance between the quarks is shorter, and hence leading to a larger contribution of coulomb term to their mass than in unequal mass mesons ( $c \bar{u}, c \bar{s}$, and $b \bar{u}, b \bar{s}$ respectively). However, since it 

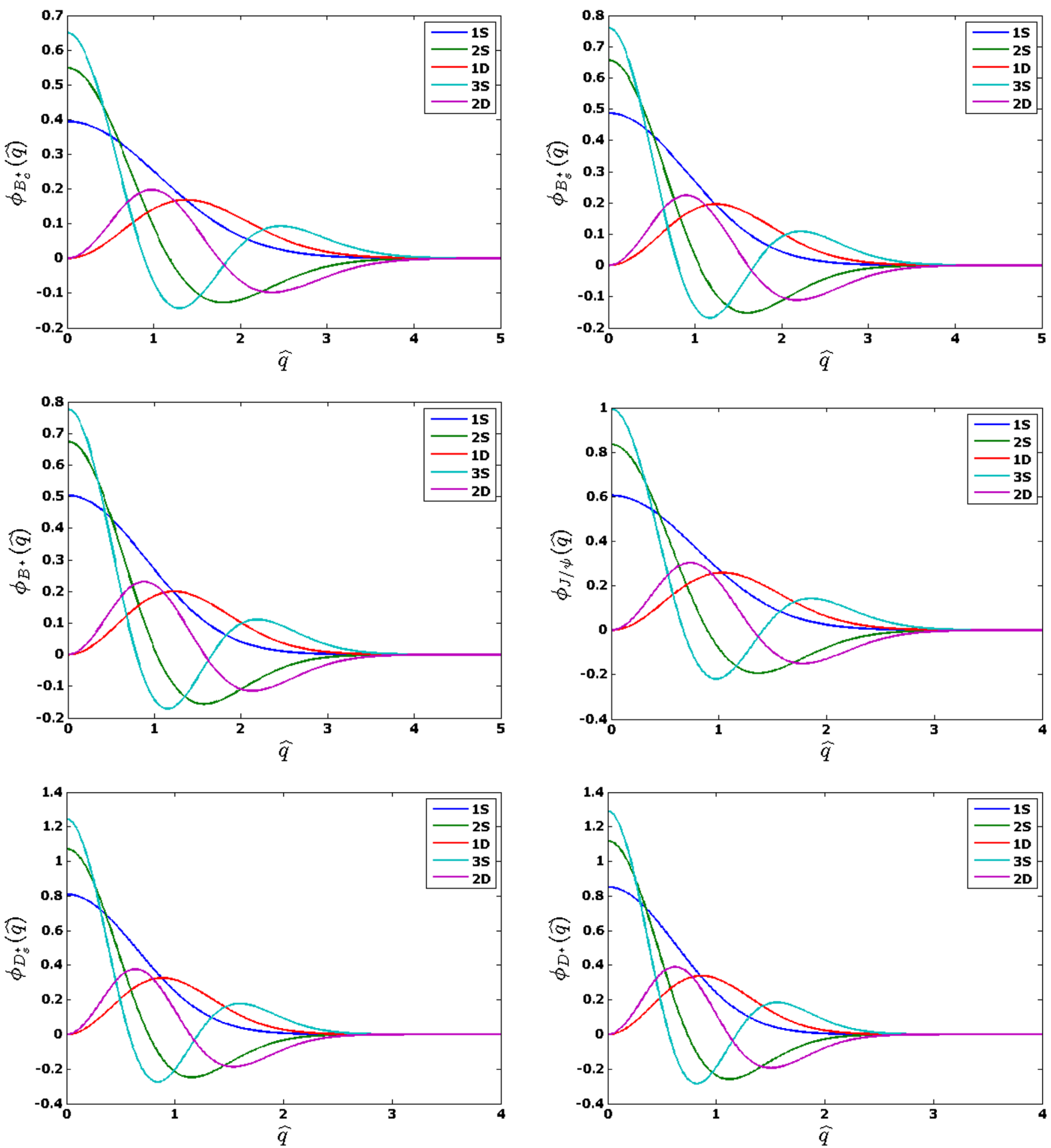

FIG. 4. Plots of wave functions for states $(1 S, \ldots, 3 S)$ Vs $\hat{q}$ (in Gev.) for vector mesons, such as $B_{c}^{*}, B_{s}^{*}, B^{*}, J / \Psi, D_{s}^{*}$, and $D^{*}$, respectively.

is further seen in Fig. 1, the confining potential for $c \bar{c}$ systems is more attractive (deeper) than the $b \bar{u}$, and $b \bar{s}$ systems in Fig. 1, this may lead to a stronger attractive coulomb force in $J / \Psi$, leading to a comparable contribution of $V_{\text {coul }}$ to its mass as $B^{*}$, and $B_{s}^{*}$. However for $B_{c}^{*}$, the contribution of $V_{\text {coul }}$ to its mass is larger than the corresponding contribution from $J / \Psi$ (which is in perfect agreement with their plots of wave functions), meaning thereby that $B_{c}$ is more tightly bound than $J / \Psi$, which is in agreement with the arguments given in [3].

We now calculate the leptonic decays of pseudoscalar and vector $Q \bar{q}$ mesons in the next sections. 
TABLE III. Masss spectra of ground and excited states of pseudoscalar $0^{-+}$quarkonia (in GeV) (with the percentage contribution of the OGE to meson mass) along with data and results of other models.

\begin{tabular}{|c|c|c|c|c|c|c|c|}
\hline & BSE-CIA & $\%$ OGE & Expt. [21] & QCD Sum Rule & PM & Lattice QCD [50] & RQM \\
\hline$M_{B_{c}\left(1 S_{0}\right)}$ & 6.2720 & $8.31 \%$ & $6.2749 \pm 0.0008$ & $6.253[55]$ & $6.349[49]$ & $6.280 \pm 0.030 \pm 0.190$ & $6.270[51]$ \\
\hline$M_{B_{c}\left(2 S_{0}\right)}$ & 6.7241 & $7.03 \%$ & & $6.863[55]$ & 6.821 [49] & $6.960 \pm 0.080$ & $6.835[51]$ \\
\hline$M_{B_{c}\left(3 S_{0}\right)}$ & 7.1968 & $5.98 \%$ & & & $7.175[49]$ & & $7.193[51]$ \\
\hline$M_{B_{c}\left(4 S_{0}\right)}$ & 7.6751 & $5.14 \%$ & & & & & \\
\hline$M_{B_{c}\left(5 S_{0}\right)}$ & 8.1499 & $4.46 \%$ & & & & & \\
\hline$M_{B_{s}\left(1 S_{0}\right)}$ & 5.3643 & $6.43 \%$ & $5.3668 \pm 0.00019$ & $5.488 \pm 0.076[56]$ & $5.367[52]$ & & $5.372[53]$ \\
\hline$M_{B_{s}\left(2 S_{0}\right)}$ & 5.7225 & $5.50 \%$ & & & $6.003[52]$ & & $5.976[53]$ \\
\hline$M_{B_{s}\left(3 S_{0}\right)}$ & 6.1496 & $4.64 \%$ & & & $6.556[52]$ & & $6.467[53]$ \\
\hline$M_{B_{s}\left(4 S_{0}\right)}$ & 6.6115 & $3.91 \%$ & & & $7.071[52]$ & & \\
\hline$M_{B_{s}\left(5 S_{0}\right)}$ & 7.0836 & $3.32 \%$ & & & $7.565[52]$ & & \\
\hline$M_{B\left(1 S_{0}\right)}$ & 5.2763 & $6.69 \%$ & $5.279 \pm 0.00014$ & $5.259 \pm 0.109[56]$ & $5.287[52]$ & & $5.280[53]$ \\
\hline$M_{B\left(2 S_{0}\right)}$ & 5.6206 & $5.75 \%$ & & & $5.926[52]$ & & $5.890[53]$ \\
\hline$M_{B\left(3 S_{0}\right)}$ & 6.0409 & $4.84 \%$ & & & $6.492[52]$ & & $6.379[53]$ \\
\hline$M_{B\left(4 S_{0}\right)}$ & 6.5001 & $4.07 \%$ & & & $7.027[52]$ & & \\
\hline$M_{B\left(5 S_{0}\right)}$ & 6.9714 & $3.45 \%$ & & & $7.538[52]$ & & \\
\hline$M_{D_{s}\left(1 S_{0}\right)}$ & 2.0541 & $1.14 \%$ & $1.9683 \pm 0.00007$ & & $1.9686[54]$ & & $1.969[53]$ \\
\hline$M_{D_{s}\left(2 S_{0}\right)}$ & 2.6358 & $0.61 \%$ & & & $2.6333[54]$ & & $2.688[53]$ \\
\hline$M_{D_{s}\left(3 S_{0}\right)}$ & 3.1891 & $0.39 \%$ & & & & & $3.129[53]$ \\
\hline$M_{D_{s}\left(4 S_{0}\right)}$ & 3.6947 & $0.27 \%$ & & & & & \\
\hline$M_{D\left(1 S_{0}\right)}$ & 1.9565 & $1.29 \%$ & $1.8648 \pm 00005$ & $1.972 \pm 0.094[56]$ & $1.8696[54]$ & & $1.871[53]$ \\
\hline$M_{D\left(2 S_{0}\right)}$ & 2.5288 & $0.68 \%$ & & & $2.5235[54]$ & & $2.581[53]$ \\
\hline$M_{D\left(3 S_{0}\right)}$ & 3.0800 & $0.42 \%$ & & & & & $3.062[53]$ \\
\hline$M_{D\left(4 S_{0}\right)}$ & 3.5821 & $0.29 \%$ & & & & & \\
\hline$M_{\eta_{c}\left(1 S_{0}\right)}$ & 3.0004 & $4.62 \%$ & $2.9839 \pm 0.0005$ & $3.11 \pm 0.52[57]$ & $2.980[58]$ & $3.292[8]$ & $2.981[18]$ \\
\hline$M_{\eta_{c}\left(2 S_{0}\right)}$ & 3.5934 & $2.98 \%$ & $3.6376 \pm 0.0012$ & & $3.600[58]$ & $4.240[8]$ & $3.635[18]$ \\
\hline$M_{\eta_{c}\left(3 S_{0}\right)}$ & 4.1433 & $2.11 \%$ & & & $4.060[58]$ & & $3.986[18]$ \\
\hline$M_{\eta_{c}\left(4 S_{0}\right)}$ & 4.6565 & $1.60 \%$ & & & $4.4554[58]$ & & $4.401[18]$ \\
\hline$M_{\eta_{c}\left(5 S_{0}\right)}$ & 5.1381 & $1.27 \%$ & & & & & \\
\hline
\end{tabular}

TABLE IV. Mass spectra of ground and excited states of vector $1^{--}$quarkonia (in $\mathrm{GeV}$ ) (with the percentage contribution of the OGE to meson mass) along with data and results of other models.

\begin{tabular}{|c|c|c|c|c|c|c|c|}
\hline & BSE-CIA & $\%$ OGE & Expt. [21] & BSE-SDE & PM & Lattice QCD & RQM \\
\hline$M_{B_{c}^{*}\left(1 S_{1}\right)}$ & 6.3514 & $9.73 \%$ & & $6.308[35]$ & $6.373[49]$ & $6.321 \pm 0.020[50]$ & $6.332[51]$ \\
\hline$M_{B_{c}^{*}\left(2 S_{1}\right)}$ & 6.8033 & $8.24 \%$ & & & $6.855[49]$ & $6.990 \pm 0.080[50]$ & $6.881[51]$ \\
\hline$M_{B_{c}^{*}(1 D)}$ & 7.0086 & $11.23 \%$ & & & & & $7.072[51]$ \\
\hline$M_{B_{c}^{*}\left(3 S_{1}\right)}$ & 7.2737 & $7.02 \%$ & & & $7.210[49]$ & & $7.235[51]$ \\
\hline$M_{B_{c}^{*}(2 D)}$ & 7.4729 & $9.60 \%$ & & & & & \\
\hline$M_{B_{c}^{*}\left(4 S_{1}\right)}$ & 7.7487 & $6.04 \%$ & & & & & \\
\hline$M_{B_{c}^{*}(3 D)}$ & 7.9398 & $8.30 \%$ & & & & & \\
\hline$M_{B_{c}^{*}\left(5 S_{1}\right)}$ & 8.2201 & $5.25 \%$ & & & & & \\
\hline$M_{B_{c}^{*}(4 D)}$ & 8.4025 & $7.25 \%$ & & & & & \\
\hline$M_{B_{s}^{*}\left(1 S_{1}\right)}$ & 5.4153 & $7.59 \%$ & $5.4154_{-0.0015}^{+0.0014}$ & $5.4130[35]$ & $5.413[52]$ & & $5.414[53]$ \\
\hline$M_{B_{s}^{*}\left(2 S_{1}\right)}$ & 5.7775 & $6.49 \%$ & & & $6.029[52]$ & & $5.992[53]$ \\
\hline$M_{B_{s}^{*}(1 D)}$ & 6.1111 & $12.14 \%$ & & & $6.119[52]$ & & $6.209[53]$ \\
\hline$M_{B_{s}^{*}\left(3 S_{1}\right)}$ & 6.2045 & $5.48 \%$ & & & $6.575[52]$ & & $6.475[53]$ \\
\hline
\end{tabular}


TABLE IV. (Continued)

\begin{tabular}{|c|c|c|c|c|c|c|c|}
\hline & BSE-CIA & $\%$ OGE & Expt. [21] & BSE-SDE & PM & Lattice QCD & RQM \\
\hline$M_{B_{s}^{*}(2 D)}$ & 6.5360 & $10.30 \%$ & & & $6.642[52]$ & & $6.629[53]$ \\
\hline$M_{B_{s}^{*}\left(4 S_{1}\right)}$ & 6.6640 & $4.62 \%$ & & & $7.087[52]$ & & \\
\hline$M_{B_{s}^{*}(3 D)}$ & 6.9824 & $8.77 \%$ & & & $7.139[52]$ & & \\
\hline$M_{B_{s}^{*}\left(5 S_{1}\right)}$ & 7.1330 & $3.94 \%$ & & & $7.579[52]$ & & \\
\hline$M_{B_{s}^{*}(4 D)}$ & 7.4344 & $7.54 \%$ & & & & & \\
\hline$M_{B^{*}\left(1 S_{1}\right)}$ & 5.3283 & $7.90 \%$ & $5.325 \pm 0.0004[20]$ & $5.325[35]$ & $5.323[52]$ & & $5.325[53]$ \\
\hline$M_{B^{*}\left(2 S_{1}\right)}$ & 5.6774 & $6.77 \%$ & & & $5.947[52]$ & & $5.848[53]$ \\
\hline$M_{B^{*}(1 D)}$ & 6.0196 & $12.61 \%$ & & & $6.016[52]$ & & $6.005[53]$ \\
\hline$M_{B^{*}\left(3 S_{1}\right)}$ & 6.0976 & $5.71 \%$ & & & $6.508[52]$ & & $6.136[53]$ \\
\hline$M_{B^{*}(2 D)}$ & 6.4386 & $10.68 \%$ & & & $6.562[52]$ & & $6.248[53]$ \\
\hline$M_{B^{*}\left(4 S_{1}\right)}$ & 6.5543 & $4.81 \%$ & & & $7.039[52]$ & & \\
\hline$M_{B^{*}(3 D)}$ & 6.8817 & $9.08 \%$ & & & $7.081[52]$ & & \\
\hline$M_{B^{*}\left(5 S_{1}\right)}$ & 7.0223 & $4.09 \%$ & & & $7.549[52]$ & & \\
\hline$M_{B^{*}(4 D)}$ & 7.3316 & $7.80 \%$ & & & & & \\
\hline$M_{D_{s}^{*}\left(1 S_{1}\right)}$ & 2.1153 & $4.32 \%$ & $2.1122 \pm 0.0004$ & $2.157[35]$ & $2.1123[54]$ & & $2.111[53]$ \\
\hline$M_{D_{s}^{*}\left(2 S_{1}\right)}$ & 2.6855 & $2.38 \%$ & $2.7083_{-0.0034}^{+0.0040}$ & & $2.7164[54]$ & & $2.731[53]$ \\
\hline$M_{D_{s}^{*}(1 D)}$ & 2.9243 & $10.12 \%$ & & & $2.9145[54]$ & & $2.919[53]$ \\
\hline$M_{D_{s}^{*}\left(3 S_{1}\right)}$ & 3.2289 & $1.52 \%$ & & & $3.2626[54]$ & & $3.242[53]$ \\
\hline$M_{D_{s}^{*}(2 D)}$ & 3.4266 & $6.80 \%$ & & & $3.3928[54]$ & & $3.383[53]$ \\
\hline$M_{D_{s}^{*}\left(4 S_{1}\right)}$ & 3.7280 & $1.08 \%$ & & & & & \\
\hline$M_{D_{s}^{*}(3 D)}$ & 3.8966 & $4.97 \%$ & & & & & \\
\hline$M_{D^{*}\left(1 S_{1}\right)}$ & 2.0221 & $4.84 \%$ & $2.010 \pm 0.00005$ & $2.068[35]$ & $2.0104[54]$ & & $2.010[53]$ \\
\hline$M_{D^{*}\left(2 S_{1}\right)}$ & 2.5821 & $2.63 \%$ & & & $2.6062[54]$ & & $2.632[53]$ \\
\hline$M_{D^{*}(1 D)}$ & 2.8056 & $10.06 \%$ & & & $2.8029[54]$ & & $2.788[53]$ \\
\hline$M_{D^{*}\left(3 S_{1}\right)}$ & 3.1222 & $1.65 \%$ & & & $3.1484[54]$ & & $3.096[53]$ \\
\hline$M_{D^{*}(2 D)}$ & 3.3053 & $6.67 \%$ & & & $3.2818[54]$ & & $3.228[53]$ \\
\hline$M_{D^{*}\left(4 S_{1}\right)}$ & 3.6171 & $1.16 \%$ & & & & & \\
\hline$M_{D^{*}(3 D)}$ & 3.7721 & $4.82 \%$ & & & & & \\
\hline$M_{J / \psi\left(1 S_{1}\right)}$ & 3.0970 & $7.84 \%$ & $3.0969 \pm 0.000006$ & & $3.0969[58]$ & 3.099 [59] & $3.096[18]$ \\
\hline$M_{\psi\left(2 S_{1}\right)}$ & 3.6744 & $5.14 \%$ & $3.6861 \pm 0.000025$ & & $3.6890[58]$ & $3.653[59]$ & $3.685[18]$ \\
\hline$M_{\psi(1 D)}$ & 3.7716 & $7.62 \%$ & $3.773 \pm 0.00033$ & & & & $3.783[18]$ \\
\hline$M_{\psi\left(3 S_{1}\right)}$ & 4.2133 & $3.69 \%$ & $4.039 \pm 0.001$ & & $4.1407[58]$ & $4.099[59]$ & $4.039[18]$ \\
\hline$M_{\psi(2 D)}$ & 4.2979 & $5.53 \%$ & $4.191 \pm 0.005$ & & & & $4.150[18]$ \\
\hline$M_{\psi\left(4 S_{1}\right)}$ & 4.7182 & $2.82 \%$ & $4.421 \pm 0.004$ & & $4.5320[58]$ & & $4.427[18]$ \\
\hline$M_{\psi(3 D)}$ & 4.7933 & $4.26 \%$ & & & & & $4.507[18]$ \\
\hline$M_{\psi\left(5 S_{1}\right)}$ & 5.1935 & $2.24 \%$ & & & 4.8841 [58] & & $4.837[18]$ \\
\hline$M_{\psi(4 D)}$ & 5.2613 & $3.41 \%$ & & & & & $4.857[18]$ \\
\hline
\end{tabular}

\section{LEPTONIC DECAYS OF PSEUDOSCALAR HEAVY-LIGHT QUARKONIA}

The leptonic decays of pseudoscalar quarkonia proceed through the coupling of the quark-antiquark loop to the axial vector current. The leptonic decay constants, $f_{P}$ are defined as,

$$
\text { if } P_{P} P_{\mu} \equiv\left\langle 0\left|\bar{Q} i \gamma_{\mu} \gamma_{5} Q\right| P\right\rangle \text {. }
$$

The decay constants can be expressed through the quark-loop integral as,

$$
f_{P} P_{\mu}=\sqrt{3} \int \frac{d^{4} q}{(2 \pi)^{4}} \operatorname{Tr}\left[\Psi^{P}(P, q) i \gamma_{\mu} \gamma_{5}\right]
$$

Making use of the fact that the 4D volume integral can be expressed as, $d^{4} q=d^{3} \hat{q} M d \sigma$, where, $M d \sigma$ is the longitudinal component of internal momentum $q_{\mu}$, and making use of the fact that under covariant instantaneous Ansatz (CIA), the 3D BS wave function, $\psi_{P}(\hat{q})$ can be expressed as,

$$
\psi_{P}(\hat{q})=i \int \frac{M d \sigma}{2 \pi} \Psi(P, q)
$$

we can express the decay constant, $f_{P}$ as a $3 \mathrm{D}$ integral $[41,60]$, 
TABLE V. Leptonic decay constants, $f_{P}$ of ground state (1S) and excited state $(2 \mathrm{~S})$ and $(3 \mathrm{~S})$ of heavy-light pseudoscalar mesons (in $\mathrm{GeV}$.) in the present calculation (BSE-CIA) along with experimental data, and their masses in other models.

\begin{tabular}{|c|c|c|c|c|c|c|}
\hline & BSE-CIA & Expt. & BSE-SDE & QCD SR & Latt. QCD & Rel. PM [61] \\
\hline$f_{\eta_{c}(1 S)}$ & 0.3282 & $0.335 \pm 0.075[25]$ & & $0.260 \pm 0.075[57]$ & $0.3928[62]$ & \\
\hline$f_{\eta_{c}(2 S)}$ & 0.2363 & & & & & \\
\hline$f_{\eta_{c}(3 S)}$ & 0.1959 & & & & & \\
\hline$f_{B_{c}(1 S)}$ & 0.4635 & & & $0.400 \pm 0.015[63]$ & $0.434 \pm 0.015[6]$ & \\
\hline$f_{B_{c}(2 S)}$ & 0.3611 & & & & & \\
\hline$f_{B_{c}(3 S)}$ & 0.3110 & & & & & \\
\hline$f_{B_{s}(1 S)}$ & 0.2589 & & & $0.195[64]$ & & $0.2288 \pm 0.0069$ \\
\hline$f_{B_{s}(2 S)}$ & 0.2087 & & & & & \\
\hline$f_{B_{s}(3 S)}$ & 0.1789 & & & & & \\
\hline$f_{B(1 S)}$ & 0.2192 & & & $0.1915 \pm 0.0073[65]$ & & $0.198 \pm 0.014$ \\
\hline$f_{B(2 S)}$ & 0.1779 & & & & & \\
\hline$f_{B(3 S)}$ & 0.1517 & & & & & \\
\hline$f_{D_{s}(1 S)}$ & 0.2033 & $0.2546 \pm 0.0059[66]$ & $0.228[35]$ & & $0.241 \pm 0.0003[67]$ & $0.256 \pm 0.026$ \\
\hline$f_{D_{s}(2 S)}$ & 0.1360 & & & & & \\
\hline$f_{D_{s}(3 S)}$ & 0.1079 & & & & & \\
\hline$f_{D(1 S)}$ & 0.1751 & $0.2067 \pm 0.0089[68]$ & $0.267[35]$ & & $0.207 \pm 0.0004[67]$ & $0.208 \pm 0.021$ \\
\hline$f_{D(2 S)}$ & 0.1145 & & & & & \\
\hline$f_{D(3 S)}$ & 0.0889 & & & & & \\
\hline
\end{tabular}

$$
f_{P}=\sqrt{3} N_{P} \int \frac{d^{3} \hat{q}}{(2 \pi)^{3}} \operatorname{Tr}\left[i \gamma_{5} \gamma_{\mu} \psi_{P}(\hat{q})\right]
$$

Here, the full 3D BS wave function, $\psi_{P}(\hat{q})$ can be taken from Eq. (A5) in the Appendix, where $\phi_{1}(\hat{q})$, and $\phi_{2}(\hat{q})$ satisfy two identical decoupled equations, Eq. (16), leading to $\phi_{1}(\hat{q})=\phi_{2}(\hat{q})\left(=\phi_{P}(\hat{q})\right)$, which is expressed as,

$$
\begin{aligned}
\psi_{P}(\hat{q})= & N_{P}\left[M-i \not P+\frac{i M\left(\omega_{1}-\omega_{2}\right)}{\omega_{1} m_{2}+m_{1} \omega_{2}} \hat{q}\right. \\
& \left.+\frac{\left(m_{1}+m_{2}\right)}{\omega_{1} \omega_{2}+m_{1} m_{2}-\hat{q}^{2}} \not \hat{\phi}\right] \gamma_{5} \phi_{P}(\hat{q})
\end{aligned}
$$

Putting the above expression for $\psi_{P}$ in Eq. (34), and evaluating trace over the gamma matrices on the right side of the equation, we get,

$f_{P} P_{\mu}=4 \sqrt{3} \int \frac{d^{3} q}{(2 \pi)^{3}}\left[P_{\mu}-\frac{M\left(\omega_{1}-\omega_{2}\right)}{m_{1} \omega_{1}+m_{2} \omega_{2}} \hat{q}_{\mu}\right] \phi_{P}(\hat{q})$.

To evaluate $f_{P}$, we multiply both sides of the above equation by $\frac{P_{\mu}}{M^{2}}$, and making use of the fact that $\hat{q} \cdot P=0$, we get,

$$
f_{P}=4 \sqrt{3} N_{P} \int \frac{d^{3} \hat{q}}{(2 \pi)^{3}} \phi_{P}(\hat{q}),
$$

where the 3D wave functions, $\phi_{P}(\hat{q})$ for pseudoscalar $Q \bar{q}$ are the unnormalized states in Eqs. (19), and $N_{P}$ is the $4 \mathrm{D}$ BS normalizer obtained through the current conservation condition,

$$
\begin{aligned}
2 i P_{\mu}= & \int \frac{d^{4} q}{(2 \pi)^{4}} \operatorname{Tr}\left\{\bar{\Psi}(P, q)\left[\frac{\partial}{\partial P_{\mu}} S_{F}^{-1}\left(p_{1}\right)\right]\right. \\
& \left.\times \Psi(P, q) S_{F}^{-1}\left(-p_{2}\right)\right\}+(1 \rightleftharpoons 2),
\end{aligned}
$$

where $\Psi_{P}(\hat{q})$ is the 4D BS wave function, while the adjoint BS wave function, $\bar{\Psi}(P, q)=\gamma_{4} \Psi^{\dagger}(P, q) \gamma_{4}$.

Leptonic decay constants of $0^{-+}$quarkonia are given in Table V along with data and results of other models.

\section{LEPTONIC DECAYS OF HEAVY-LIGHT VECTOR QUARKONIA}

Leptonic decay constant of vector mesons is expressed through the coupling of the quark-antiquark loop to the vector current as,

$$
f_{V} M \epsilon_{\mu}(P) \equiv\left\langle 0\left|\bar{Q}_{\gamma_{\mu}} Q\right| V(P)\right\rangle .
$$

Following a similar procedure as in the case of pseudoscalar mesons, we can express the leptonic decays of vector quarkonia, $f_{V}$ as a quark-loop integral,

$$
f_{V} M \epsilon_{\mu}=\sqrt{3} \int \frac{d^{3} \hat{q}}{(2 \pi)^{3}} \operatorname{Tr}\left[\psi^{V}(\hat{q}) \gamma_{\mu}\right] .
$$

Here $\psi^{V}(\hat{q})$ is the full 3D wave function for vector mesons that can be taken from Eq. (A8), where $\chi_{1}(\hat{q})$, and $\chi_{2}(\hat{q})$ satisfy two identical decoupled equations, Eq. (23), leading to $\chi_{1}(\hat{q})=\chi_{2}(\hat{q})\left(=\phi_{V}(\hat{q})\right)$, which is expressed as, 


$$
\begin{aligned}
\psi_{V}(\hat{q})= & N_{V}\left[i M \not+\hat{q} \cdot \varepsilon \frac{M\left(m_{1}+m_{2}\right)}{\omega_{1} \omega_{2}+m_{1} m_{2}-\hat{q}^{2}}+\not \not P\right. \\
& \left.+\frac{i\left(\omega_{1}-\omega_{2}\right)}{2\left(\omega_{1} m_{2}+m_{1} \omega_{2}\right)}(\not \partial \phi \hat{\phi}+\hat{q} \cdot \varepsilon \not P)\right] \phi_{V}(\hat{q})
\end{aligned}
$$

Putting Eq. (41) in Eq. (40), and evaluating trace over the gamma matrices on the RHS, and multiplying both sides of the resulting equation by the polarization vector, $\epsilon_{\mu}$ of vector meson, and making use of the fact that, $P . \epsilon=0$, and the $3 \mathrm{D}$ reduction through Eq. (33), we get the leptonic decay constant of vector mesons as,

$$
f_{V}=4 \sqrt{3} N_{V} \int \frac{d^{3} \hat{q}}{(2 \pi)^{3}} \phi_{V}(\hat{q})
$$

where the 4D BS normalizer, $N_{V}$ can be obtained from the current conservation condition in Eq. (38), and following a similar procedure as in the case of pseudoscalar quarkonia with the expression for BS normalizer, $N_{V}$ given in the Appendix.

The leptonic decay constants of heavy-light vector mesons are given in Table VI.

TABLE VI. Leptonic decay constants, $f_{V}$ of ground state (1S) and excited state $(2 \mathrm{~S}), \ldots,(3 \mathrm{~S})$ of heavy-light vector mesons (in $\mathrm{GeV}$.) in the present calculation (BSE-CIA) along with experimental data, and their masses in other models.

\begin{tabular}{lcccc}
\hline \hline & BSE - CIA & Expt. & BSE [16] & RQM [71] \\
\hline$f_{B c *(1 S)}$ & 0.5171 & & $0.418 \pm 0.024$ & \\
$f_{B c *(2 S)}$ & 0.4292 & & $0.331 \pm 0.021$ & \\
$f_{B c *(1 D)}$ & 0.5441 & & & \\
$f_{B c *(3 S)}$ & 0.3927 & & & \\
$f_{B s *(1 S)}$ & 0.3097 & & $0.272 \pm 0.020$ & 0.214 \\
$f_{B s *(2 S)}$ & 0.2585 & & $0.246 \pm 0.013$ & \\
$f_{B s *(1 D)}$ & 0.2945 & & & \\
$f_{B s *(3 S)}$ & 0.2292 & & & \\
$f_{B *(1 S)}$ & 0.2473 & & $0.238 \pm 0.018$ & 0.195 \\
$f_{B *(2 S)}$ & 0.2109 & & $0.221 \pm 0.014$ & \\
$f_{B *(1 D)}$ & 0.2359 & & & \\
$f_{B *(3 S)}$ & 0.1877 & & & \\
$f_{D s *(1 S)}$ & 0.2698 & $0.227[72]$ & $0.375 \pm 0.024$ & 0.335 \\
$f_{D s *(2 S)}$ & 0.2026 & & $0.312 \pm 0.017$ & \\
$f_{D s *(1 D)}$ & 0.2483 & & & \\
$f_{D s *(3 S)}$ & 0.1749 & & & \\
$f_{D *(1 S)}$ & 0.2251 & $0.196[72]$ & $0.339 \pm 0.022$ & 0.315 \\
$f_{D *(2 S)}$ & 0.1680 & & $0.289 \pm 0.016$ & \\
$f_{D *(1 D)}$ & 0.2035 & & & \\
$f_{D *(3 S)}$ & 0.1441 & & & \\
$f_{J / \psi(1 S)}$ & 0.4599 & $0.411[20]$ & & \\
$f_{\psi(2 S)}$ & 0.3256 & $0.279[20]$ & & \\
$f_{\psi(1 D)}$ & 0.2687 & $0.210[20]$ & & \\
$f_{\psi(3 S)}$ & 0.2218 & & & \\
\hline \hline & & & & \\
\hline
\end{tabular}

\section{RESULTS AND DISCUSSION}

We have employed a 3D reduction of BSE (with a $4 \times 4$ representation for two-body $(q \bar{q}) \mathrm{BS}$ amplitude) under covariant instantaneous Ansatz (CIA) with an interaction kernel consisting of both the confining and one gluon exchange terms, to derive the algebraic forms of the mass spectral equations and eigen functions of heavy-light quarkonia in an approximate harmonic oscillator basis, leading to mass spectra of ground and excited states of heavy-light scalar $\left(0^{++}\right)$, pseudoscalar $\left(0^{-+}\right)$, and vector $\left(1^{--}\right)$quarkonia.

The input parameters of our model obtained by best fit to the spectra of ground states of scalar, pseudoscalar and vector $Q \bar{q}$, and $Q \bar{Q}$ quarkonia are $C_{0}=0.69$, $\omega_{0}=0.22 \mathrm{GeV}, \Lambda_{\mathrm{QCD}}=0.250 \mathrm{GeV}$, and $A_{0}=0.01$, with input quark masses $m_{u}=0.300 \mathrm{GeV}, m_{s}=0.430 \mathrm{GeV}$, $m_{c}=1.490 \mathrm{GeV}$, and $m_{b}=4.690 \mathrm{GeV}$. We further calculated the percentage contribution of short range coulomb term, $\gamma\left\langle V_{\text {coulomb }}\right\rangle$ to the mass of each meson state, which are indeed small, justifying the perturbative treatment of the Coulomb term for these states. We see that for any $J^{P C}$, the contribution of Coulomb term to meson mass for $b \bar{u}, b \bar{s}$, and $c \bar{b}$ mesons is larger than the corresponding contributions from $c \bar{u}, c \bar{s}$, and $c \bar{c}$ states, implying thereby that the former states are more tightly bound than the latter. This may be due to the fact that the heavier $b$ quark would pull the lighter $c, u, s$ quarks more strongly (a similar argument was given in [3] to suggest that the $c$ quark in $B_{c}$ moves faster than in $J / \Psi$ since it must balance the momentum of a more massive $b$ quark), and hence being more tightly bound, and with a larger contribution of Coulomb term to their mass than the lighter $c \bar{u}, c \bar{s}$, and $c \bar{c}$ states. Further, as seen from Tables II, III, and IV, for a given meson, as we go from its ground state $(n=1)$ to its excited $(n=2,3,4, \ldots)$ states, the contribution of Coulomb term to its mass keeps decreasing. Due to this, the ground states of mesons are more tightly bound than their excited states. This is similar to the feature seen in atoms, with the ground states being more tightly bound than the excited states.

We wish to point out that this above feature is also supported by the plots of analytic forms [that are derived analytically in Eqs. (12), (19), and (27)] of the long distance (nonperturbative) wave functions of $0^{++}, 0^{-+}$, and $1^{--}$ respectively, as a function of the internal momentum, $\hat{q}$ in Figs. 2-4. These plots show that the wave functions, $\phi(n S)$, $\phi(n D)$, and $\phi(n P)$ have $(n-1)$ nodes, which is a general feature of bound quantum mechanical systems. As mentioned in Sec. V, for $Q \bar{q}$ systems, the wave functions show a damped oscillatory behavior, with amplitude for $n S$ states (of $0^{-+}$, and $1^{--}$), and $n P$ states (of $0^{++}$), being maximum at $0 \mathrm{GeV}$ (confinement region), and falling gradually with increase in $\hat{q}$, and finally becoming zero. An interesting feature of these plots is that as the mass of the meson, $M$ increases, $\phi(\hat{q}) \rightarrow 0$ at a higher value of $|\hat{q}|$. This implies that the wave functions of heavier mass $Q \bar{q}$ systems (such 
as $\left.B_{c}, B_{c}^{*}, B_{s}, B_{s}^{*}, B, B^{*}\right)$ extend to a much shorter distance than the wave functions of $\left(\chi_{c 0}, \eta_{c}, J / \Psi, D, D^{*}, D_{s}, D_{s}^{*}\right)$, implying thereby that the heavier mesons $\left(B_{c}, B_{c}^{*}, B_{s}, \ldots\right)$ are more tightly bound than the comparatively lighter mesons $\left(\chi_{c 0}, \eta_{c}, J / \Psi, D, D^{*}, D_{s}, \ldots\right)$. Due to this, one can expect a larger contribution of the Coulomb term to the mass of $c \bar{b}, b \bar{u}$, and $b \bar{s}$ states than the lighter $c \bar{c}, c \bar{s}$, and $c \bar{u}$ states, though there are some exceptions as mentioned in detail in Sec. V. Thus the algebraic forms of 3D hadronic BS wave functions can not only provide information about the long range nonperturbative physics, but also tell us the shortest distance to which they can extend to in a hadron. Hence these hadronic BS wave functions are physically reasonable, and build a connection between the long range nonperturbative physics, and the short range perturbative physics.

These wave functions for heavy-light mesons so derived, are then used to calculate the leptonic decay constants for heavy-light pseudoscalar and vector mesons as a test of the wave functions derived and the BSE framework employed. As stated earlier, the partitioning of relativistic internal momentum $q$ comes from the Wightmann-Garding definitions $\hat{m}_{1,2}$ of masses of individual quarks. The 3D reduction through covariant instantaneous Ansatz (CIA) employed by us does make our formulation relativistically covariant, but it is not be Poincare covariant, since our results depend on the momentum partitioning parameters. In a Poincare covariant framework [69,70], the numerical results for the amplitudes and masses are independent of the choice of momentum partitioning parameters.

In this work, we make use of the exact treatment of the spin structure $\left(\gamma_{\mu} \otimes \gamma_{\mu}\right)$ in the interaction kernel, in contrast to the approximate treatment of the same in our previous works $[41,42])$. In so doing we do away with the approximation of taking the leading Dirac structures in the structure of 4D BS wave function, $\Psi(P, q)$, which is a substantial improvement over our previous works. We thus first derive analytically the mass spectral equation using only the confining part of the interaction kernel for $Q \bar{q}$ systems, and calculate the algebraic forms of the wave functions. Then treating this mass spectral equation as the unperturbed equation, we introduce the one-gluon-exchange (OGE) perturbatively, and obtain the mass spectra for various states of $0^{++}, 0^{-+}$, and $1^{--}$, treating the wave functions derived above as the unperturbed wave functions.

As mentioned earlier, in our works, we are not only interested in studying the mass spectrum of hadrons, which no doubt is an important element to study dynamics of hadrons, but also the hadronic wave functions that play an important role in the calculation of decay constants, form factors, structure functions etc. for $Q \bar{Q}$, and $Q \bar{q}$ hadrons. As mentioned above, these hadronic Bethe-Salpeter wave functions calculated algebraically in this work provide a lot of information about the long distance nonperturbative physics. And since these quarkonia are involved in a number of reactions which are of great importance for study of Cabibbo-Kobayashi-Maskawa (CKM) matrix and $C P$ violation, the wave functions calculated analytically by us can lead to studies on a number of processes involving $Q \bar{Q}$, and $Q \bar{q}$ states. In this work, we have used these algebraic forms of wave functions to calculate the leptonic decay constants of pseudoscalar and vector $Q \bar{q}$ quarkonia in Tables V and VI respectively. We will be further using these wave functions of ground and excited states of heavylight quarkonia to evaluate the various transition processes involving heavy-light scalar, pseudoscalar, and vector quarkonia as future work.

\section{ACKNOWLEDGEMENTS}

We thank Chandigarh University and Addis Ababa University where this work was carried out. One of us (E. G.) would like to thank Woldia University for support for his Doctoral programme at Addis Ababa University. E. G. would also like to acknowledge the hospitality at Chandigarh University for his research visit under Swedish International Development Agency (SIDA). We thank the unknown referee for his valuable comments that led to the improvement of the article. We thank Dr. B. Kavita for discussions, and encouragement.

\section{APPENDIX}

\section{Scalar mesons}

For scalar mesons, the Salpeter equations $\psi^{+-}(\hat{q})=$ $\psi^{-+}(\hat{q})=0$ in Eq. (2), are used to obtain the constraint relations between the scalar functions for unequal mass mesons as

$$
\begin{aligned}
& f_{1}(\hat{q})=\frac{-\left(m_{1}+m_{2}\right) \hat{q}^{2}}{M\left(\omega_{1} \omega_{2}+m_{1} m_{2}-\hat{q}^{2}\right)} f_{3}(\hat{q}), \\
& f_{2}(\hat{q})=\frac{2\left(\omega_{2}-\omega_{1}\right) \hat{q}^{2}}{M\left(\omega_{1} m_{2}+m_{1} \omega_{2}\right)} f_{4}(\hat{q}) .
\end{aligned}
$$

The BS-wave function for scalar mesons in Eq. (7) with the help of these constraint relations can be rewritten in terms of only two independent scalar functions $\left(f_{1}\right.$ (or $f_{3}$ ) and $\left.f_{4}\right)$ as

$$
\psi^{S}(\hat{q})=\left(\frac{-\left(m_{1}+m_{2}\right) \hat{q}^{2}}{\left(\omega_{1} \omega_{2}+m_{1} m_{2}-\hat{q}^{2}\right)}-i \hat{q}\right) f_{3}(\hat{q})-2\left(\frac{i\left(\omega_{2}-\omega_{1}\right) \hat{q}^{2} \not P}{M\left(\omega_{1} m_{2}+m_{1} \omega_{2}\right)}+\frac{\not \hat{q}_{q}}{M}\right) f_{4}(\hat{q})
$$


The coupled equations that result from the first two Salpeter equations (with use of confining interaction alone) are

$$
\begin{aligned}
{[M} & \left.-\omega_{1}-\omega_{2}\right]\left[\frac{2 \omega_{1} \omega_{2}\left(m_{1}+m_{2}\right)}{\omega_{1} \omega_{2}+m_{1} m_{2}-\hat{q}^{2}} f_{3}(\hat{q})+\frac{4 \omega_{1} \omega_{2}\left(m_{1}+m_{2}\right)}{\omega_{1} m_{2}+m_{1} \omega_{2}} f_{4}(\hat{q})\right]=-\frac{1}{\hat{q}^{2}} \int \frac{d^{3} \hat{q}^{\prime}}{(2 \pi)^{3}} V_{c}\left(\hat{q}, \hat{q}^{\prime}\right) \\
& \times\left[\left(\frac{4\left(\omega_{1} \omega_{2}-m_{1} m_{2}+\hat{q}^{2}\right)\left(m_{1}+m_{2}\right) \hat{q}^{\prime 2}}{\omega_{1} \omega_{2}+m_{1} m_{2}-\hat{q}^{\prime 2}}+2\left(m_{1}+m_{2}\right) \hat{q} \cdot \hat{q}^{\prime}\right) f_{3}\left(\hat{q}^{\prime}\right)\right. \\
& \left.-\left(\frac{4\left(\omega_{1} m_{2}-m_{1} \omega_{2}\right)\left(\omega_{2}-\omega_{1}\right) \hat{q}^{\prime 2}}{\omega_{1} m_{2}+m_{1} \omega_{2}}\right) f_{4}\left(\hat{q}^{\prime}\right)\right] \\
{[M} & \left.+\omega_{1}+\omega_{2}\right]\left[\frac{-2 \omega_{1} \omega_{2}\left(m_{1}+m_{2}\right)}{\omega_{1} \omega_{2}+m_{1} m_{2}-\hat{q}^{2}} f_{3}(\hat{q})+\frac{4 \omega_{1} \omega_{2}\left(m_{1}+m_{2}\right)}{\omega_{1} m_{2}+m_{1} \omega_{2}} f_{4}(\hat{q})\right]=-\frac{1}{\hat{q}^{2}} \int \frac{d^{3} \hat{q}^{\prime}}{(2 \pi)^{3}} V_{c}\left(\hat{q}, \hat{q}^{\prime}\right) \\
& \times\left[\left(\frac{4\left(\omega_{1} \omega_{2}-m_{1} m_{2}+\hat{q}^{2}\right)\left(m_{1}+m_{2}\right) \hat{q}^{\prime 2}}{\omega_{1} \omega_{2}+m_{1} m_{2}-\hat{q}^{\prime 2}}+2\left(m_{1}+m_{2}\right) \hat{q} \cdot \hat{q}^{\prime}\right) f_{3}\left(\hat{q}^{\prime}\right)\right. \\
& \left.+4\left(\frac{\left(\omega_{1} m_{2}-m_{1} \omega_{2}\right)\left(\omega_{2}-\omega_{1}\right) \hat{q}^{\prime 2}}{\omega_{1} m_{2}+m_{1} \omega_{2}}\right) f_{4}\left(\hat{q}^{\prime}\right)\right]
\end{aligned}
$$

To decouple these equations, we follow the same procedure in $[41,42]$, where we first add them. Then we subtract the second equation from the first equation. For a kernel that can be expressed as $V_{c}\left(\hat{q}-\hat{q}^{\prime}\right)=$ $\bar{V}_{c}(\hat{q}) \delta^{3}\left(\hat{q}-\hat{q}^{\prime}\right)$, we get two algebraic equations which are still coupled. Then from one of the two equations so obtained, we eliminate $f_{3}(\hat{q})$ in terms of $f_{4}(\hat{q})$, and plug this expression for $f_{3}(\hat{q})$ in the second equation of the coupled set so obtained to get a decoupled equation in $f_{4}(\hat{q})$. Similarly, we eliminate $f_{4}(\hat{q})$ from the second equation of the set of coupled algebraic equations in terms of $f_{3}(\hat{q})$, and plug it into the first equation to get a decoupled equation entirely in $f_{3}(\hat{q})$, which reduces to two identical decoupled equations, one entirely in $f_{3}(\hat{q})$, and the other that is entirely in $f_{4}(\hat{q})$.

\section{Pseudoscalar mesons}

We use the last two Salpeter equations in Eq. (2) to find the constraints on the components of the wave function as

$$
\phi_{4}(\hat{q})=\frac{M\left(m_{1}+m_{2}\right)}{\omega_{1} \omega_{2}+m_{1} m_{2}-\hat{q}^{2}} \phi_{2}(\hat{q}), \quad \phi_{3}(\hat{q})=\frac{M\left(\omega_{1}-\omega_{2}\right)}{\omega_{1} m_{2}+m_{1} \omega_{2}} \phi_{1}(\hat{q}),
$$

Plugging Eq. (A4) into Eq. (15), we rewrite the wave function for pseudoscalar mesons as

$$
\psi^{P}(\hat{q})=\left[\left(M+\frac{i M\left(\omega_{1}-\omega_{2}\right)}{\omega_{1} m_{2}+m_{1} \omega_{2}} \hat{q}\right) \phi_{1}(\hat{q})+\left(-i \not P+\frac{\left(m_{1}+m_{2}\right)}{\omega_{1} \omega_{2}+m_{1} m_{2}-\hat{q}^{2}} \not \hat{q} \hat{q}\right) \phi_{2}(\hat{q})\right] \gamma_{5} .
$$

Using the above constraint relations, the first two Salpeter equations can be expressed as coupled integral equations as:

$$
\begin{aligned}
& {\left[M-\omega_{1}-\omega_{2}\right]\left[\left(\frac{\left(m_{2} \omega_{2}\right) \omega_{1}^{2}+\left(m_{1} \omega_{1}\right) \omega_{2}^{2}}{\omega_{1} m_{2}+m_{1} \omega_{2}}\right) \phi_{1}(\hat{q})+\left(\frac{\left(m_{2} \omega_{2}\right) \omega_{1}^{2}+\left(m_{1} \omega_{1}\right) \omega_{2}^{2}}{\omega_{1} \omega_{2}+m_{1} m_{2}-\hat{q}^{2}}\right) \phi_{2}(\hat{q})\right]} \\
& =\int \frac{d^{3} \hat{q}^{\prime}}{(2 \pi)^{3}} V_{c}\left(\hat{q}, \hat{q}^{\prime}\right)\left[\left(-2\left(\omega_{1} \omega_{2}+m_{1} m_{2}+\hat{q}^{2}\right)-\frac{\left(m_{1}-m_{2}\right)\left(\omega_{1}-\omega_{2}\right)}{\omega_{1} m_{2}+m_{1} \omega_{2}} \hat{q} \cdot \hat{q}^{\prime}\right) \phi_{1}\left(\hat{q}^{\prime}\right)\right. \\
& \left.\quad+\left(\omega_{1} m_{2}+m_{1} \omega_{2}\right) \phi_{2}\left(\hat{q}^{\prime}\right)\right] \\
& {\left[M+\omega_{1}+\omega_{2}\right]\left[\left(\frac{\left(m_{2} \omega_{2}\right) \omega_{1}^{2}+\left(m_{1} \omega_{1}\right) \omega_{2}^{2}}{\omega_{1} m_{2}+m_{1} \omega_{2}}\right) \phi_{1}(\hat{q})-\left(\frac{\left(m_{2} \omega_{2}\right) \omega_{1}^{2}+\left(m_{1} \omega_{1}\right) \omega_{2}^{2}}{\omega_{1} \omega_{2}+m_{1} m_{2}-\hat{q}^{2}}\right) \phi_{2}(\hat{q})\right]} \\
& =-\int \frac{d^{3} \hat{q}^{\prime}}{(2 \pi)^{3}} V_{c}\left(\hat{q}, \hat{q}^{\prime}\right)\left[\left(-2\left(\omega_{1} \omega_{2}+m_{1} m_{2}+\hat{q}^{2}\right)-\frac{\left(m_{1}-m_{2}\right)\left(\omega_{1}-\omega_{2}\right)}{\omega_{1} m_{2}+m_{1} \omega_{2}} \hat{q} \cdot \hat{q}^{\prime}\right) \phi_{1}\left(\hat{q}^{\prime}\right)\right. \\
& \left.\quad-\left(\omega_{1} m_{2}+m_{1} \omega_{2}\right) \phi_{2}\left(\hat{q}^{\prime}\right)\right]
\end{aligned}
$$


These coupled equations are again decoupled following the same procedure as in scalar meson case.

\section{Vector mesons}

The constraint equations on the components of the wave functions $(\chi \mathrm{s})$ can be obtained using the last two Salpeter equations of (2) as

$$
\begin{aligned}
& \chi_{5}(\hat{q})=\frac{M\left(m_{1}+m_{2}\right)}{\omega_{1} \omega_{2}+m_{1} m_{2}-\hat{q}^{2}} \chi_{1}(\hat{q}), \quad \chi_{4}(\hat{q})=-\frac{M\left(\omega_{1}+\omega_{2}\right)}{2\left(\omega_{1} m_{2}+m_{1} \omega_{2}\right)} \chi_{2}(\hat{q}) \\
& \chi_{3}(\hat{q})=\chi_{6}(\hat{q})=0 .
\end{aligned}
$$

Substituting Eq. (A7) into Eq. (22), the wave function for vector mesons can be rewritten as

$$
\psi^{V}(\hat{q})=\left(i M \phi+\hat{q} . \epsilon \frac{M\left(m_{1}+m_{2}\right)}{\omega_{1} \omega_{2}+m_{1} m_{2}-\hat{q}^{2}}\right) \chi_{1}(\hat{q})+\left(\not \not P+\frac{i\left(\omega_{1}+\omega_{2}\right)}{2\left(\omega_{1} m_{2}+m_{1} \omega_{2}\right)}(\not P \phi \hat{\phi}+\hat{q} . \epsilon \not \partial)\right) \chi_{2}(\hat{q})
$$

Using the first two Salpeter equations, we obtain the coupled integral equations of vector mesons (with confining interaction alone) as

$$
\begin{aligned}
& {\left[M-\omega_{1}-\omega_{2}\right] \hat{q} \cdot \epsilon\left[\frac{2 \omega_{1} \omega_{2}\left(m_{1}+m_{2}\right)}{\omega_{1} \omega_{2}+m_{1} m_{2}-\hat{q}^{2}} \chi_{1}(\hat{q})-\frac{2 \omega_{1} m_{2}\left(\omega_{1}+\omega_{2}\right)}{\omega_{1} m_{2}+m_{1} \omega_{2}} \chi_{2}(\hat{q})\right]=\int \frac{d^{3} \hat{q}^{\prime}}{(2 \pi)^{3}} V_{c}\left(\hat{q}, \hat{q}^{\prime}\right)} \\
& \times\left[-\left(2 \hat{q} \cdot \epsilon\left(m_{1}+m_{2}\right)+4 \hat{q}^{\prime} \cdot \epsilon\left(m_{1}+m_{2}\right) \frac{\left(\omega_{1} \omega_{2}-m_{1} m_{2}+\hat{q}^{2}\right)}{\omega_{1} \omega_{2}+m_{1} m_{2}-\hat{q}^{\prime 2}}\right) \chi_{1}\left(\hat{q}^{\prime}\right)\right. \\
& \left.+\left(2 \hat{q}^{\prime} \cdot \epsilon\left(\omega_{1}+\omega_{2}\right) \frac{\left(\omega_{1} m_{2}-m_{1} \omega_{2}\right)}{\omega_{1} m_{2}+m_{1} \omega_{2}}\right) \chi_{2}\left(\hat{q}^{\prime}\right)\right] \\
& {\left[M+\omega_{1}+\omega_{2}\right] \hat{q} \cdot \epsilon\left[\frac{2 \omega_{1} \omega_{2}\left(m_{1}+m_{2}\right)}{\omega_{1} \omega_{2}+m_{1} m_{2}-\hat{q}^{2}} \chi_{1}(\hat{q})+\frac{2 \omega_{1} m_{2}\left(\omega_{1}+\omega_{2}\right)}{\omega_{1} m_{2}+m_{1} \omega_{2}} \chi_{2}(\hat{q})\right]=-\int \frac{d^{3} \hat{q}^{\prime}}{(2 \pi)^{3}} V_{c}\left(\hat{q}, \hat{q}^{\prime}\right)} \\
& \times\left[-\left(2 \hat{q} \cdot \epsilon\left(m_{1}+m_{2}\right)+4 \hat{q}^{\prime} \cdot \epsilon\left(m_{1}+m_{2}\right) \frac{\left(\omega_{1} \omega_{2}-m_{1} m_{2}+\hat{q}^{2}\right)}{\left.\omega_{1} \omega_{2}+m_{1} m_{2}-\hat{q}^{\prime 2}\right) \chi_{1}\left(\hat{q}^{\prime}\right)}\right.\right. \\
& \left.-\left(2 \hat{q}^{\prime} \cdot \epsilon\left(\omega_{1}+\omega_{2}\right) \frac{\left(\omega_{1} m_{2}-m_{1} \omega_{2}\right)}{\omega_{1} m_{2}+m_{1} \omega_{2}}\right) \chi_{2}\left(\hat{q}^{\prime}\right)\right] .
\end{aligned}
$$

\section{BS normalizers}

(a) Pseudoscalar mesons: We make use of the fact that in the inverse propagators, $S_{F}^{-1}\left(p_{1,2}\right)$ of the two quarks, their momenta are expressed as, $p_{1,2}=\hat{m}_{1,2} P \pm q$, and the $4 \mathrm{D}$ volume element, $d^{4} q=d^{3} \hat{q} M d \sigma$. Integrating over $M d \sigma$, and making use of the 3D form of BS wave function, $\psi(\hat{q})$, in Eq. (35), evaluating trace over the gamma matrices, and multiplying both sides of the equation by $P_{\mu}$, we get,

$$
\begin{aligned}
N_{P}^{-2}= & \int \frac{d^{3} \hat{q}}{(2 \pi)^{3}} \frac{\phi_{P}^{2}(\hat{q})}{m_{1}}\left[\frac{4 M^{2} \hat{m}_{1} \hat{m}_{2}\left(\omega_{1}-\omega_{2}\right)^{2} \hat{q}^{2}}{\left(\omega_{1} m_{2}+m_{1} \omega_{2}\right)^{2}}+\frac{4 M^{2} \hat{m}_{1} \hat{m}_{2}\left(m_{1}+m_{2}\right)^{2} \hat{q}^{2}}{\left(\omega_{1} \omega_{2}+m_{1} m_{2}-\hat{q}^{2}\right)^{2}}\right. \\
& +\frac{8 M \hat{m}_{1}\left(m_{1}+m_{2}\right) m_{2}\left(\omega_{1}-\omega_{2}\right) \hat{q}^{2}}{\left(\omega_{1} m_{2}+m_{1} \omega_{2}\right)\left(\omega_{1} \omega_{2}+m_{1} m_{2}-\hat{q}^{2}\right)}+\frac{8 M \hat{m}_{1}\left(m_{1}+m_{2}\right) \hat{q}^{2}}{\left(\omega_{1} \omega_{2}+m_{1} m_{2}-\hat{q}^{2}\right)} \\
& \left.+\frac{8 M \hat{m}_{1}\left(\omega_{1}-\omega_{2}\right) \hat{q}^{2}}{\left(\omega_{1} m_{2}+m_{1} \omega_{2}\right)}\right]+(1 \rightleftharpoons 2),
\end{aligned}
$$

(b) Vector mesons: Following the same procedure as in the case of pseudoscalar meson, the BS normalizer for vector meson is expressed as, 


$$
\begin{aligned}
N_{V}^{-2}= & \int \frac{d^{3} \hat{q}}{(2 \pi)^{3}} \frac{\phi_{V}^{2}(\hat{q})}{m_{1}}\left[-\frac{2 M^{2} \hat{m}_{1} \hat{m}_{2}\left(\omega_{1}+\omega_{2}\right)^{2}(\hat{q} \cdot \epsilon)^{2}}{\left(\omega_{1} m_{2}+m_{1} \omega_{2}\right)^{2}}-\frac{8 M^{2} \hat{m}_{1} \hat{m}_{2}\left(m_{1}+m_{2}\right)^{2}(\hat{q} \cdot \epsilon)^{2}}{\left(\omega_{1} \omega_{2}+m_{1} m_{2}-\hat{q}^{2}\right)^{2}}\right. \\
& +\frac{16 M \hat{m}_{1}\left(m_{1}+m_{2}\right) m_{2}\left(\omega_{1}+\omega_{2}\right)(\hat{q} \cdot \epsilon)^{2}}{\left(\omega_{1} m_{2}+m_{1} \omega_{2}\right)\left(\omega_{1} \omega_{2}+m_{1} m_{2}-\hat{q}^{2}\right)}+\frac{8 M \hat{m}_{1}\left(\omega_{1}+\omega_{2}\right)(\hat{q} \cdot \epsilon)^{2}}{\left(\omega_{1} m_{2}+m_{1} \omega_{2}\right)} \\
& \left.+\frac{16 M \hat{m}_{1}\left(m_{1}+m_{2}\right)(\hat{q} \cdot \epsilon)^{2}}{\left(\omega_{1} \omega_{2}+m_{1} m_{2}-\hat{q}^{2}\right)}\right]+(1 \rightleftharpoons 2),
\end{aligned}
$$

where $\varepsilon_{\mu}$ is the polarization vector of the vector meson of mass, $M$, and momentum, $P$, and is normalized as, $\varepsilon_{\mu} \varepsilon_{\nu}=\frac{1}{3}\left(\delta_{\mu, \nu}+\frac{P_{\mu} P_{\nu}}{M^{2}}\right)$.

[1] N. Brambilla, A. Pineda, J. Soto, and A. Vairo, Rev. Mod. Phys. 77, 1423 (2005).

[2] M. A. Shifman, A. I. Vainshtein, and V. I. Zakharov, Nucl. Phys. B147, 385 (1979).

[3] E. J. Eichten and C. Quigg, Phys. Rev. D 99, 054025 (2019).

[4] S. Godfrey and N. Isgur, Phys. Rev. D 32, 189 (1985).

[5] D. Ebert, R. N. Faustov, and V. O. Galkin, Mod. Phys. Lett. A 18, 601 (2003).

[6] B. Colquhoun, C. T. S. Davies, R. J. Dowdall, J. Kettle, J. Koponen, G. P. Lepage, A. T. Lytle (HPQCD Collaboration), Phys. Rev. D 91, 114509 (2015).

[7] G. S. Bali, K. Schiling, and A. Wachter, Phys. Rev. D 56, 2566 (1997).

[8] T. Burch, C. DeTar, M. Di Pierro, A. X. El-Khadra, E. D. Freeland, S. Gottlieb, A. S. Kronfeld, L. Levkova, P. B. Mackenzie, and J. N. Simone (Fermilab and MILC Collaborations), Phys. Rev. D 81, 034508 (2010).

[9] A. N. Mitra and B. M. Sodermark, Nucl. Phys. A695, 328 (2001).

[10] R. Alkofer and L. Von Smekal, Phys. Rep. 353, 281 (2001).

[11] A. N. Mitra and S. Bhatnagar, Int. J. Mod. Phys. A 07, 121 (1992).

[12] H. J. Munczek and P. Jain, Phys. Rev. D 46, 438 (1992); P. Jain and H. J. Munczek, Phys. Rev. D 48, 5403 (1993).

[13] S. Bhatnagar, J. Mahecha, and Y. Mengesha, Phys. Rev. D 90, 014034 (2014).

[14] S. Bhatnagar and S.-Y. Li, J. Phys. G 32, 949 (2006).

[15] S. Bhatnagar, S.-Y. Li, and J. Mahecha, Int. J. Mod. Phys. E 20, 1437 (2011).

[16] Z. K. Geng, T. Wang, Y. Jiang, G. Li, X.-Z. Tan, and G.-L. Wang, Phys. Rev. D 99, 013006 (2019); G. L. Wang, Phys. Lett. B 633, 492 (2006).

[17] M. Neubert, Phys. Rep. 245, 259 (1994).

[18] D. Ebert, R. N. Faustov, and V. O. Galkin, Phys. At. Nucl. 76, 1554 (2013); Eur. Phys. J. C 71, 1825 (2011).

[19] J. Gasser and H. Leutwyler, Ann. Phys. (N.Y.) 158, 142 (1984).

[20] K. A. Olive et al. (Particle Data Group), Chin. Phys. C 38, 090001 (2014).
[21] M. Tanabashi et al. (PDG Collaboration), Phys. Rev. D 98, 030001 (2018).

[22] B. Auger et al. (BABAR Collaboration), Phys. Rev. Lett. 103, 161801 (2009).

[23] K. M. Ecklund et al. (CLEO Collaboration), Phys. Rev. D 78, 091501 (2008).

[24] K. F. Chen et al. (Belle Collaboration), Phys. Rev. D 82, 091106(R) (2010).

[25] K. W. Edwards et al. (CLEO Collaboration), Phys. Rev. Lett. 86, 30 (2001).

[26] F. K. Guo and U.-G. Meisner, Phys. Rev. D 86, 091501 (2012).

[27] S. L. Olsen, Phys. Rev. D 91, 057501 (2015).

[28] M. Ablikim et al. (BESIII Collaboration), Phys. Rev. Lett. 110, 252001 (2013).

[29] B. Aubert et al. (BABAR Collaboration), Phys. Rev. Lett. 95, 142001 (2005).

[30] C. Z. Yuan et al. (Belle Collaboration), Phys. Rev. Lett. 99, 182004 (2007).

[31] S. K. Choi et al. (Belle Collaboration), Phys. Rev. Lett. 91, 262001 (2003).

[32] L. Chang, M. Chen, and Y. X. Liu, arXiv:1904.00399.

[33] J. C. Su, Prog. Theor. Phys. 114, 391 (2005).

[34] M. Ding, K. Raya, A. Bashir, D. Binosi, L. Chang, M. Chen, and C. D. Roberts, Phys. Rev. D 99, 014014 (2019).

[35] M. A. Bedolla, E. Santopinto, and L. X. Gutierrez-Guerrero, EPJ Web Conf. 192, 00039 (2018); arXiv:1810.04391.

[36] V. A. Karmanov, J. Karbonell, and H. Sazdjian, EPJ Web Conf. 204, 01014 (2019).

[37] T. Fredrico, G. Salme, and M. Viviani, Phys. Rev. D 89, 016010 (2014).

[38] J. Carbonell, T. Fredrico, and V. A. Karmonov, Phys. Lett. B 769, 418 (2017).

[39] J. He, Eur. Phys. J. C 79, 393 (2019).

[40] Z. Y. Wang, J. J. Qi, and X. H. Guo, Adv. High Energy Phys. 2019, 7576254 (2019).

[41] H. Negash and S. Bhatnagar, Int. J. Mod. Phys. E 25, 1650059 (2016).

[42] S. Bhatnagar and L. Alemu, Phys. Rev. D 97, 034021 (2018).

[43] E. Mengesha and S. Bhatnagar, Int. J. Mod. Phys. E 22, 1350046 (2013). 
[44] H. Negash and S. Bhatnagar, Int. J. Mod. Phys. E 24, 1550030 (2015).

[45] H. Negash and S. Bhatnagar, Adv. High Energy Phys. 2017, 7306825 (2017).

[46] C. B. Yang and X. Cai, Phys. Rev. D 51, 6332 (1995).

[47] A. Sternbeck, M. Leutnant, and G. Eichmann, Proc. Sci., LATTICE2018 (2019) 068.

[48] C. H. L. Smith, Ann. Phys. (N.Y.) 53, 521 (1969).

[49] A. K. Rai and P. C. Vinodkumar, Pramana J. Phys. 66, 953 (2006).

[50] C. T. H. Davies, K. Hornbostel, G. P. Lepage, A. J. Lidsey, J. Shigemitsu, and J.H. Sloan, Phys. Lett. B 382, 131 (1996).

[51] D. Ebert, R. N. Faustov, and V. O. Galkin, Phys. Rev. D 67, 014027 (2003).

[52] V. Kher, N. Devlani, and A. K. Rai, Chin. Phys. C 41, 093101 (2017).

[53] D. Ebert, R. Faustov, and V. Galkin, Eur. Phys. J. C 66, 197 (2010).

[54] B. Patel and P.C. Vinodkumar, arXiv:0908.2212v1; M. Shah, B. Patel, and P. C. Vinodkumar, Phys. Rev. D 90 , 014009 (2014).

[55] S. S. Gershtein, V. V. Kiselev, A. K. Likhoded, and A. V. Tkabladze, Phys. Rev. D 51, 3613 (1995); Usp. Fiz. Nauk 165, 3 (1995) [Phys. Usp. 38, 1 (1995)].

[56] H. Mutuk, Adv. High Energy Phys. 2018, 8095653 (2018).

[57] E. Veli Veliev, K. Azizi, H. Sundu, and N. Aksit, J. Phys. G 39, 015002 (2012).
[58] Bhagyesh, K. B. V. Kumar, and A. P. Monterio, J. Phys. G 38, 085001 (2011).

[59] T. Kawanai and S. Sasaki, AIP Conf. Proc. 1701, 050022 (2016).

[60] J. K. He and Y. D. Yang, Nucl. Phys. B943, 114627 (2019).

[61] M.-Z. Yang, Eur. Phys. J. C 72, 1880 (2012).

[62] C. McNielle, C. T. H. Davies, E. Follana, K. Hornbostel, G. P. Lepage (HPQCD Collaboration), Phys. Rev. D 86, 074503 (2012).

[63] V. V. Kiselev, arXiv:hep-ph/0211021v2.

[64] Z. G. Wang, W. M. Yang, and S. L. Wan, Nucl. Phys. A744, 156 (2004).

[65] W. Lucha, D. Melikhov, and S. Simula, Proc. Sci., EPSHEP2013 (2013) 021.

[66] B. I. Eisenstein et al. (CLEO Collaboration), Phys. Rev. D 78, 052003 (2008).

[67] E. Follana, C. T. H. Davies, G. P. Lepage, and J. Shigemitsu, Phys. Rev. Lett. 100, 062002 (2008).

[68] J. Beringer et al. (Particle Data Group), Phys. Rev. D 86, 010001 (2012).

[69] T. Hilger, C. Popovici, M. Gomez-Rocha, and A. Krassnigg, Phys. Rev. D 91, 034013 (2015).

[70] T. Hilger, M. Gomez-Rocha, and A. Krassnigg, Phys. Rev. D 91, 114004 (2015).

[71] D. Ebert, R. N. Faustov, and V. O. Galkin, Mod. Phys. Lett. A 17, 803 (2002).

[72] C. Patrignani et al. (Particle Data Group), Chin. Phys. C 40, 100001 (2016). 